Journal of physical oceanography, Vol. 23, No. 11, pp. 2373-2391, 1993

\title{
Numerical Simulation of Flow around a Tall Isolated Seamount. Part II: Resonant Generation of Trapped Waves
}

\author{
Dale B. Haidvogel, ${ }^{*}$ Aike Beckmann, ${ }^{* *}$ David C. Chapman, ${ }^{\dagger}$ and Ray-Qing Lin ${ }^{\ddagger}$ \\ * Institute of Marine and Coastal Sciences, Rutgers University, New Brunswick, New Jersey \\ **Institut fïr Meereskunde, Abteilung Theoretische Ozeanographie, Kiel, Germany \\ ${ }^{+}$Woods Hole Oceanographic Institution, Woods Hole, Massachusetts \\ ${ }^{\ddagger}$ Ocean Ice Branch, Goddard Space Flight Center, Greenbelt, Maryland
}

(Manuscript received 28 January 1992, in final form 2 March 1993)

\begin{abstract}
A sigma-coordinate, primitive equation ocean circulation model is used to explore the problem of the resonant generation of trapped waves about a tall, circular, isolated seamount by an incident oscillatory barotropic current. The numerical solutions are used to extend prior studies into the fully nonlinear regime, and in particular to quantify and interpret the occurrence of residual circulation. Specific attention is also devoted to the dependence of the resonance and rectification mechanisms on stratification, forcing frequency, and choice of subgrid-scale viscous closure.

Resonantly generated trapped waves of significant amplitude are found to occur broadly in parameter space; a precise match between the frequency of the imposed incident current and the frequency of the trapped free wave is not necessary to produce substantial excitation of the trapped wave. The maximum amplification factors produced in these numerical solutions, $\mathrm{O}(100)$ times the strength of the incident current, are consistent with previous studies.

In the presence of nonlinear advection, strong residual currents are produced. The time-mean circulation about the seamount is dominated by a strong bottom-intensified, anticyclonic circulation closely trapped to the seamount. Maximum local time-mean current amplitudes are found to be as large as $37 \%$ of the magnitude of the propagating waves. In addition to the strong anticyclonic residual flow, there is a weaker secondary circulation in the vertical-radial plane characterized by downwelling over the top of the seamount at all depths. Maximum vertical downwelling rates of several tens of meters per day occur at the summit of the seamount. The vertical mass flux implied by this systematic downwelling is balanced by a slow radial flux of mass directed outward along the flanks of the seamount.

Time-mean budgets for the radial and azimuthal components of momentum show that horizontal eddy fluxes of momentum are responsible for transporting net radial and azimuthal momentum from the far field to the upper flanks of the seamount. There, Coriolis and pressure gradient forces provide the dominant balances in the radial direction. However, the Coriolis force and viscous effects provide the primary balance for the azimuthal component.
\end{abstract}

\section{Introduction}

Observational studies have now documented enhanced subinertial currents over isolated topographic features at a variety of locations in the world's oceans. Amplified currents of diurnal frequency have, for example, been identified over Rockall Bank (Huthnance 1974), the Yermak Plateau (Hunkins 1986), and Fieberling Guyot (Genin et al. 1989). A recent illustration of this phenomenon has emerged from the results of TOPO, a multi-institutional program supported by the U.S. Office of Naval Research to study the physical, chemical, and biological properties of oceanic flow near

Corresponding author address: Dr. Dale B. Haidvogel, Institute of Marine and Coastal Studies, Rutgers University, P.O. Box 231, New Brunswick, New Jersey 08903-0231. abrupt topography. The specific focus of TOPO is Fieberling Guyot, a tall, isolated seamount located in the eastern North Pacific Ocean. Current meter records obtained as part of a pilot project of TOPO from a telemetering mooring set atop Fieberling have recently been shown to be dominated by diurnal frequency fluctuations of large amplitude $\left(0.2-0.4 \mathrm{~m} \mathrm{~s}^{-1}\right)$ that exhibit some of the properties of freely propagating seamount-trapped waves (Eriksen 1991). Significant mean circulation $\left[\mathrm{O}(0.1) \mathrm{m} \mathrm{s}^{-1}\right]$ was also detected about the seamount, suggesting efficient nonlinear rectification of the diurnal currents.

A considerable body of theoretical evidence suggests that these oscillations represent freely propagating subinertial frequency waves resonantly excited by the diurnal oscillating tide. The resonant generation of seamount-trapped waves by excitation of oscillatory ambient currents has been explored recently in the 
barotropic and stratified limits by Chapman (1989) and Brink (1990), respectively. Both studies clearly demonstrate substantial amplification of a freely propagating, subinertial trapped wave if the forcing frequency is sufficiently close to the natural frequency of the free wave. In neither model was amplification found to depend in a sensitive way on seamount size or on an exact match between forcing and free-wave frequency. Consequently, both authors conclude that resonantly generated, topographically trapped motions may well be a common occurrence in nature.

Though the topographic rectification of tidal currents has been most often explored in the context of an infinitely long ridge or shelf topography, such studies are clearly pertinent to the issue of mean flow generation about an isolated seamount. Recent theoretical studies of tidal rectification include the work of Wright and Loder (1985) in the unstratified, finite amplitude topography limit, and the analysis by Maas and Zimmerman $(1989 a, b)$ for stratified flow over small amplitude topography. These studies demonstrate broad sets of circumstances under which substantial tidal rectification can occur and identify several characteristic features of the rectified flow including a bottomintensified structure and net cross-isobath flow directed radially outward from the topography. However, both also emphasize the parametric sensitivity of the process, most importantly to the assumed parameterization of subgrid-scale viscous effects.

Here, we extend these prior studies into the stratified, nonlinear, finite amplitude topography limit using a three-dimensional, primitive equation ocean circulation model. The model is the semispectral sigma coordinate model (SPEM) of Haidvogel et al. (1991b), which has now been successfully applied to a variety of regional modeling studies featuring strongly sloping bottom topography-for example, to cross-shelf exchange in the coastal transition zone (Haidvogel et al. 1991a) and to the trapping of fluid parcels near isolated seamounts (Chapman and Haidvogel 1992 and 1993). For initial simplicity, we will follow the latter of these studies in considering a smooth, radially symmetric seamount, and a purely oscillatory, barotropic incident current. The additional effects of seamount roughness, and of horizontal and vertical current shears, are presently being studied for future presentation.

The plan of the paper is as follows. Section 2 describes the experimental configuration and solution procedures employed in this study. Section 3 considers the forced behavior of the linearized equations, describes the resulting amplified flow trapped to the seamount, and investigates the parametric sensitivity of the resonance process. Section 4 addresses the issue of mean flow generation in the presence of advective nonlinearity, and describes the dynamical mechanism involved. Lastly, section 5 offers concluding remarks and discussion.

\section{Experimental configuration and solution procedures}

The setting for our study is a tall circular isolated seamount in an initially resting ocean with fixed background stratification $\rho_{r}(z)$. The equations of motion and solution techniques are briefly described next. Additional detail on both may be found in the companion paper by Beckman and Haidvogel (1993; hereafter BH).

\section{a. Configuration}

The model configuration is that of a periodic $f$-plane channel; the flow is bounded by two free-slip walls ( $y$ $=0, L_{y}$ ), and is assumed to be reentrant (periodic) in the alongchannel coordinate $\left(0 \leqslant x \leqslant L_{x}\right)$. A Gaussian seamount is placed in the center of the channel with a topographic profile given by

$$
h(x, y)=H_{o}-H e^{\left(-\left(x^{2}+y^{2}\right) / L^{2}\right)},
$$

where $H_{o}=4500 \mathrm{~m}, L=25 \mathrm{~km}$, and $H=4050 \mathrm{~m}$. The external dimensions of the channel $\left(L_{x}=380 \mathrm{~km}\right.$, $L_{y}=288 \mathrm{~km}$ ) are more than ten times the radius of the seamount.

The experiments described below start with initially level isopycnals around the seamount, and a state of no motion. As our reference stratification, we prescribe either a linear profile,

$$
\rho_{r}=\rho_{o}+24-\Delta_{z} \rho(z / 4500 \mathrm{~m}),
$$

or the exponential stratification,

$$
\rho_{r}=\rho_{o}+28-\Delta_{z} \rho e^{(z / 1000 \mathrm{~m})},
$$

where $\Delta_{z} \rho$ in the units of $\mathrm{kg} \mathrm{m}^{-3}$ is the density difference between the surface and the maximum water depth, and $\rho_{o}$ is a constant reference density. The two density profiles (2) and (3) have equal buoyancy frequencies at a depth of about $1500 \mathrm{~m}$.

The forcing is introduced by varying the boundary value for the barotropic streamfunction $\psi$ at one channel wall,

$$
\psi\left(y=L_{y}, t\right)=\psi_{o} \sin \left(2 \pi t / T_{f}\right),
$$

while holding the streamfunction value fixed (zero) at the other. In the central case below, the model is forced with a sinusoidally varying transport of amplitude $\left(\psi_{0}\right.$ =) $2.6 \mathrm{~Sv}\left(1 \mathrm{~Sv} \equiv 10^{6} \mathrm{~m}^{3} \mathrm{sec}^{-1}\right)$. The forcing period $\left(T_{f}\right)$ is initially set to one day to simulate a weak diurnal tidal forcing. In the far-field, this is equivalent to an incident barotropic flow of amplitude

$$
U_{i}=\frac{\psi_{o}}{H_{o} L_{y}}=0.2 \mathrm{~cm} \mathrm{~s}^{-1} .
$$

To reduce the model's inertial wave response, the forcing is increased gradually from zero until it reaches its full strength after about 20 days. 


\section{b. Numerical solution}

The dynamical model employed is the semispectral $\sigma$-coordinate model of Haidvogel et al. (199lb). With the $\sigma$-coordinate, $\sigma=2(z / H)+1$, the nonlinear equations of motion become

$$
\begin{gathered}
h \frac{\partial u}{\partial t}+\nabla \cdot(h u \mathbf{v})-h f v \\
=-h \frac{\partial \phi}{\partial x}-h(1-\sigma) \frac{\partial}{\partial \sigma}\left(\frac{\phi}{h}\right) \frac{\partial h}{\partial x}+D_{v} \\
h \frac{\partial v}{\partial t}+\nabla \cdot(h v \mathbf{v})+h f u \\
=-\mathrm{h} \frac{\partial \phi}{\partial y}-h(1-\sigma) \frac{\partial}{\partial \sigma}\left(\frac{\phi}{h}\right) \frac{\partial h}{\partial y}+D_{v} \\
h \frac{\partial \rho}{\partial t}+\nabla \cdot(h \rho \mathbf{v})=D_{\rho} \\
\frac{\partial \phi}{\partial \sigma}=-\frac{g h}{2 \rho_{o}} \rho
\end{gathered}
$$

and

$$
\frac{\partial}{\partial x}(h u)+\frac{\partial}{\partial y}(h v)+h \frac{\partial}{\partial \sigma} \Omega=0,
$$

where $(u, v, \Omega)$ are the $(x, y, \sigma)$ components of vector velocity $\mathrm{v}, h=h(x, y)$ is the fluid depth, $\phi(x, y, \sigma, t)$ is the dynamic pressure $\left(p / \rho_{o}\right), f(x, y)=f_{o}$ is the Coriolis parameter, and $g$ is the acceleration of gravity. Lastly, the "vertical velocity" in this coordinate system is

$$
\begin{aligned}
& \Omega(x, y, \sigma, t) \\
& \quad=\frac{1}{h}\left[(1-\sigma) u \frac{\partial h}{\partial x}+(1-\sigma) v \frac{\partial h}{\partial y}+2 w\right],
\end{aligned}
$$

where $w$ is the vertical velocity component in the original Cartesian system. (For ease of interpretation, the true vertical velocity $w$ will be referred to exclusively in the results below.)

Viscous and diffusive processes in the momentum and density equations-schematically represented above by the terms $D_{u}, D_{v}$, and $D_{\rho}$-take on a variety of forms. In many experiments, a mixing of momentum along sigma surfaces is added in the form of a biharmonic operator with a constant coefficient $\nu$. A higher-order boundary condition is provided at the channel walls to ensure no net viscous gain or loss of momentum within the domain. In contrast, the majority of our numerical simulations could be conducted with $D_{\rho} \equiv 0$. (We will refer to this as the "adiabatic" limit). However, a small nonzero coefficient of biharmonic diffusivity (directed along sigma surfaces) was required in the nonlinear runs as described further below.
With so little known concerning small-scale mixing in the ocean, particularly in the neighborhood of seamounts, it would be useless to try to defend any specific frictional parameterization too strongly, and we will not attempt to do so here with our biharmonic operator (or any other). An important question of course is whether the properties of the resonantly generated waves, and the dynamical pathways that support them, are qualitatively influenced by the choice of subgridscale closure. In an attempt to answer this question, we have also experimented quite broadly with bottom friction and Rayleigh damping as alternatives to biharmonic smoothing.

Prior to numerical solution, the vertical structure of the model variables is represented using an expansion in Chebyshev polynomials, which have the advantage of rapid convergence of the vertical representation. This choice also results in increased vertical resolution at the surface and the bottom boundaries. A total of seven Chebyshev polynomials are used in the vertical expansion. In the horizontal coordinates, a centered, secondorder finite-difference approximation is adopted (an Arakawa "C" grid; Arakawa and Lamb 1977). The grid spacing varies smoothly from $4.0 \mathrm{~km}$ over the seamount to $8.0 \mathrm{~km}$ at the domain boundaries (Fig. 1).

Despite its rather natural treatment of variable bathymetry, the sigma-coordinate approach is now well known to suffer from potentially large, systematic errors in the resulting discretized pressure gradient terms. In particular, spurious accelerations of significant amplitude can be shown to result even in the presence of initially level isopycnal surfaces (see, e.g., Haney 1991). Beckmann and Haidvogel have examined the implications of these pressure gradient errors for the circulation about a tall, isolated seamount. In particular, they show that for an initially quiescent, unforced ocean with level isopycnals, and for the experimental configuration adopted here, the pressure gradient errors produce a spurious oscillating current of approximately 1 $\mathrm{cm} \mathrm{s}^{-1}$ in amplitude and roughly 0.5 days in period. In the presence of the oscillatory forcing used below, however, the erroneous current is reduced in amplitude by destructive interference with the resonantly forced trapped wave. As a result, artificial currents are at least an order of magnitude smaller than the resonantly generated waves.

\section{c. Nondimensional parameters}

Even in this rather idealized setting, there are numerous independently specifiable nondimensional parameters. Among the most important of these are

the fractional seamount height, $\delta$ :

$$
\delta=\frac{H}{H_{o}}=0.9 ;
$$




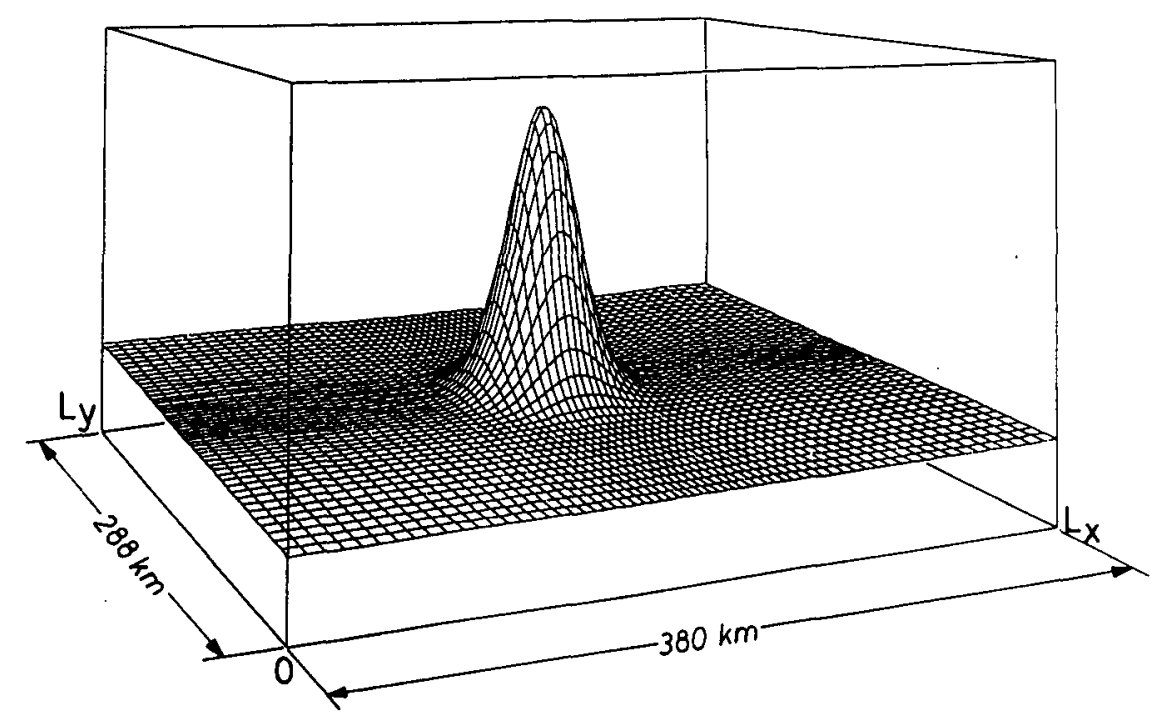

FIG. 1. Perspective plot of the seamount geometry, and the horizontal numerical grid.

the Burger number, $S$ :

$$
S=\frac{N_{o} H_{o}}{f_{o} L},
$$

where $N_{o}^{2}=-g \Delta_{z} \rho /\left(\rho_{o} H_{o}\right)$; the nondimensional forcing frequency, $\omega$ :

$$
\omega=\frac{2 \pi}{f_{o} T_{f}}
$$

and the Rossby number associated with the incident flow, $R_{i}$ :

$$
R_{i}=\frac{U_{i}}{f_{o} L}=8 \times 10^{-4} .
$$

Additional nondimensional parameters characterize the strength of the viscous terms. The dimensional viscous coefficients have been nondimensionalized as follows:

$$
\begin{aligned}
\hat{\nu} & =\nu / f_{o} L^{4}, \\
\hat{r}_{d} & =r_{d} / f_{o} H_{\min }, \\
\hat{r}_{\mathrm{Ra}} & =r_{\mathrm{Ra}} / f_{o},
\end{aligned}
$$

where $\nu, r_{d}$, and $r_{\mathrm{Ra}}$ are the dimensional values of the biharmonic lateral viscosity, the bottom drag coefficient, and the Rayleigh damping rate. Note the use of the minimum depth of the seamount as the nondimensionalizing length scale for the bottom resistance coefficient. This choice produces a great degree of similarity between the amplification curves for cases with bottom friction and those with Rayleigh damping. (The reason is that these solutions are highly bottom trapped. Hence, the effects of the Rayleigh damping term are localized at the bottom as they would be for bottom stress, though their vertical structure is dynamically determined a posteriori rather than assumed a priori.)

The parameter space represented by these nondimensional numbers is a large one. To expedite its exploration, we have fixed the values of $\delta$ and $R_{i}$ as indicated in (11) and (14), and have focused attention on variations in Burger number, the forcing frequency, and the nature and strength of the assumed subgridscale viscous processes.

\section{d. Experimental overview}

Using the experimental configuration outlined above, approximately 300 numerical solutions have been obtained to investigate the occurrence of amplified trapped waves, and to explore the parameter dependence of their generation. Two primary measures of the amplitude of the forced circulation are used: the maximum pointwise instantaneous velocity magnitude at the seamount (the "wave amplitude"), and the maximum pointwise magnitude of the time-mean circulation (the "mean flow").

Much of the initial parameter exploration was conducted using the linearized version of Eqs. (5)-(10), obtained by removing the advective terms in both horizontal momentum equations, and by linearizing the advective terms in the density equation about the reference stratification $\rho_{r}(z)$. The resulting system has the virtue that we are usually able to produce numerically stable solutions to the adiabatic $\left(D_{\rho} \equiv 0\right)$ linearized equations, so that ambiguities about the correct treatment of subgrid-scale diffusion are lessened. In addition, the linearized system has been treated by 
TABLE 1. Overview of the numerical experiments. Other parameters $\left(\delta, R_{i}\right)$ are held fixed or are varied $(S, \omega)$, as described in the text.

\begin{tabular}{|c|c|c|c|c|c|c|}
\hline Experiment & Physics & $\hat{\nu}_{u v}\left(\times 10^{-6}\right)$ & $\dot{\nu}_{\rho}\left(\times 10^{-6}\right)$ & $\hat{r}_{b}\left(\times 10^{-2}\right)$ & $\hat{r}_{\mathrm{Ra}}\left(\times 10^{-2}\right)$ & Comments \\
\hline $1 \mathrm{~A}$ & linear & 40.5 & & & & constant $N$ \\
\hline $1 \mathrm{~B}$ & linear & 128.0 & & & & constant $N$ \\
\hline $1 \mathrm{C}$ & linear & 405.0 & & & & constant $N$ \\
\hline ID & linear & 40.5 & & 5.8 & & constant $N$ \\
\hline $1 \mathrm{E}$ & linear & 40.5 & & & 5.8 & constant $N$ \\
\hline $2 \mathrm{~A}$ & linear & 128.0 & & & & exp. N (OPG \\
\hline $2 B$ & linear & 128.0 & & & & exp. N (CPG) \\
\hline $3 \mathrm{~A}$ & nonlinear & 128.0 & & & & constant $N$ \\
\hline 3B & nonlinear & 128.0 & 2.55 & & & constant $N$ \\
\hline $3 C$ & nonlinear & 1.28 & 2.55 & & 5.8 & constant $N$ \\
\hline
\end{tabular}

Chapman (1989) and Brink (1990), and thus offers the possibility of comparison with these prior studies.

Several sets of experiments will be discussed in detail in the next two sections. Table 1 provides a brief overview of their parametric attributes.

\section{Forced response of the linearized system}

\section{a. Burger number dependence}

An initial suite of experiments (1B; Table 1) was conducted to examine model response as a function of the strength of the uniform background stratification. All solutions were forced by a diurnally varying, barotropic alongchannel flow having a far-field amplitude of $2 \mathrm{~mm} \mathrm{~s}^{-1}$. The magnitude of the incident flow (2 $\mathrm{mm} \mathrm{s}^{-1}$ ) has been chosen subjectively such that the maximally resonant waves have current amplitudes of $\mathrm{O}\left(10 \mathrm{~cm} \mathrm{~s}^{-1}\right)$, comparable to the diurnal currents observed at Fieberling. Damping was provided by a weak

TABLE 2. Parameter values used in the central experiments (experiments 1B).

\begin{tabular}{|c|c|c|}
\hline Symbol & Value & Definition \\
\hline$I$ & 65 & number of points in $x$ direction \\
\hline$J$ & 49 & number of points in $y$ direction \\
\hline$K$ & 7 & number of Chebyshev polynomials \\
\hline$\rho_{0}$ & $1000 \mathrm{~kg} \mathrm{~m}^{-3}$ & constant reference density \\
\hline$g$ & $9.81 \mathrm{~m} \mathrm{~s}^{-2}$ & acceleration of gravity \\
\hline$f_{0}$ & $10^{-4} 1 \mathrm{~s}^{-1}$ & constant Coriolis parameter \\
\hline$\nu$ & $5 \times 10^{9} \mathrm{~m}^{4} \mathrm{~s}^{-1}$ & biharmonic viscosity coefficient \\
\hline$h_{\min }$ & $450 \mathrm{~m}$ & minimum water depth \\
\hline$h_{\max }$ & $4500 \mathrm{~m}$ & maximum water depth \\
\hline$L$ & $25 \mathrm{~km}$ & length scale of seamount \\
\hline$(\nabla h)_{\max }$ & $13.7 \%$ & maximum topographic slope \\
\hline$(\Delta x, \Delta y)_{\min }$ & $4.0 \mathrm{~km}$ & minimum horizontal grid spacing \\
\hline$(\Delta x, \Delta y)_{\max }$ & $8.0 \mathrm{~km}$ & maximum horizontal grid spacing \\
\hline$\Delta t$ & $432 \mathrm{~s}$ & time step \\
\hline$T_{f}$ & 1 day & forcing period \\
\hline$\psi_{0}$ & $2.6 \mathrm{~Sv}$ & forcing amplitude \\
\hline$\delta$ & 0.9 & fractional seamount height \\
\hline$S$ & $0 .-6.0$ & Burger number \\
\hline$\omega$ & 0.727 & nondimensional forcing frequency \\
\hline$R_{i}$ & $8 \times 10^{-4}$ & Rossby number of incident flow \\
\hline$\dot{\nu}$ & $1.28 \times 10^{-4}$ & nondimensional viscosity \\
\hline
\end{tabular}

biharmonic viscosity (dimensional $\nu=5 \times 10^{9}$ $\mathrm{m}^{4} \mathrm{~s}^{-1}$ ). Bottom friction, Rayleigh damping, lateral diffusion of density, and explicit vertical smoothing of fields were all absent. The strength of the constant background stratification $\left(\Delta_{z} \rho\right)$ was varied systematically to produce Burger numbers in the range $0<S$ $<6$. The parameter values used to obtain these solutions are summarized in Table 2 and the resulting wave amplitudes in Table 3.

Consistent with previous analytic and numerical results, trapped waves of significantly enhanced amplitude are generated about the seamount after only a few forcing periods. An oscillatory equilibrium is thereafter reached by about day 20 . Figure 2 shows the resonance curve for this set of experiments (wave amplitude, measured at day 25, plotted as a function of Burger number). Over this range of $S$, there exist two reso-

TABLE 3. Wave amplitudes $\left(\mathrm{cm} \mathrm{s}^{-1}\right)$ for experiments with linearized physics, forced with $2 \mathrm{~mm} \mathrm{~s}^{-1}$ at 1 -day period. An asterisk denotes a numerically unstable experiment.

\begin{tabular}{rrrrrrrr}
\hline \hline & \multicolumn{7}{c}{ Experiment } \\
\cline { 2 - 8 }$S$ & \multicolumn{1}{c}{$1 \mathrm{~A}$} & $1 \mathrm{~B}$ & $1 \mathrm{C}$ & $1 \mathrm{D}$ & $1 \mathrm{E}$ & $2 \mathrm{~A}$ & $2 \mathrm{~B}$ \\
\hline 0.0 & 3.0 & 2.7 & 2.1 & 3.4 & 2.1 & 2.7 & 2.6 \\
0.5 & 3.7 & 3.2 & 2.3 & 4.1 & 2.5 & 3.6 & 3.6 \\
1.0 & 8.1 & 6.0 & 3.4 & 8.4 & 4.5 & 10.9 & 11.2 \\
1.1 & 11.1 & 7.4 & 3.9 & 10.8 & 5.4 & 13.5 & 13.8 \\
1.2 & 15.3 & 9.6 & 4.4 & 14.7 & 6.4 & 13.0 & 12.9 \\
1.3 & 27.7 & 13.1 & 5.1 & 20.9 & 7.4 & 10.6 & 10.3 \\
1.4 & 50.1 & 17.3 & 5.6 & 23.9 & 8.1 & 8.4 & 10.4 \\
1.5 & 44.2 & 17.8 & 6.2 & 19.7 & 8.1 & 6.9 & $*$ \\
1.6 & 22.9 & 14.0 & 6.3 & 14.2 & 7.6 & 5.9 & $*$ \\
1.7 & 16.0 & 10.5 & 5.9 & 11.1 & 6.8 & 5.1 & $*$ \\
1.8 & 12.2 & 8.4 & 5.2 & 8.9 & 6.1 & 4.6 & $*$ \\
1.9 & 9.8 & 6.9 & 4.6 & 7.6 & 5.4 & 4.1 & $*$ \\
2.0 & 8.5 & 5.9 & 4.0 & 6.6 & 4.7 & 3.8 & $*$ \\
2.5 & 5.6 & 3.9 & 2.5 & 4.5 & 3.5 & 2.9 & $*$ \\
3.0 & 5.2 & 3.3 & 2.0 & 4.6 & 3.7 & 2.9 & $*$ \\
3.5 & 6.0 & 3.3 & 1.8 & 5.1 & 3.9 & 3.1 & $*$ \\
4.0 & 8.4 & 3.7 & 1.7 & 5.5 & 3.9 & $*$ & $*$ \\
4.5 & 8.8 & 3.6 & 1.6 & 5.2 & 3.5 & $*$ & $* *$ \\
5.0 & 5.4 & 2.7 & 1.4 & 3.5 & 2.7 & $*$ & $*$ \\
5.5 & 3.4 & 1.8 & 1.0 & 2.4 & 2.1 & $*$ & $*$ \\
6.0 & 3.7 & 1.8 & 1.0 & 2.6 & 1.9 & $*$ & $*$ \\
\hline & & & & & & & $*$
\end{tabular}




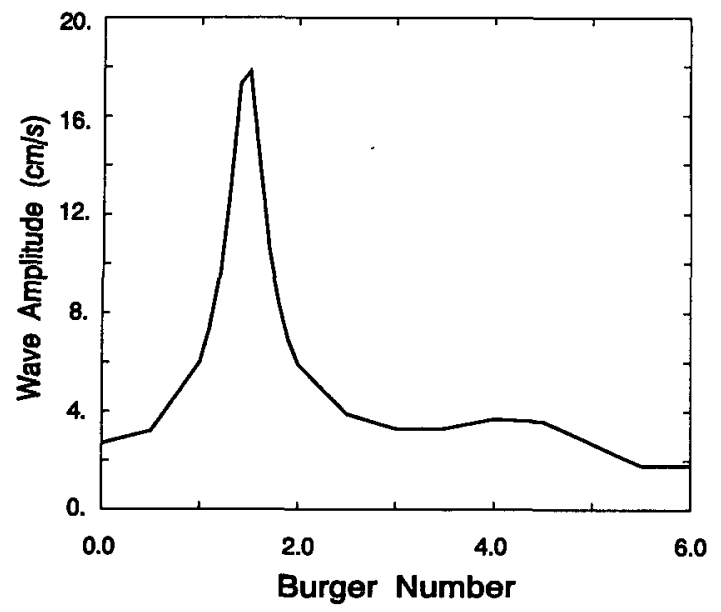

FIG. 2. Wave amplitude in centimeters per second as a function of Burger number for the central experiment. The incident flow strength is $0.2 \mathrm{~cm} \mathrm{~s}^{-1}$.

nance peaks, one near $S=1.5$ and another between $S$ $=4$ and $S=4.5$. The stronger of the two resonances is associated with a local wave amplitude of approximately $18 \mathrm{~cm} \mathrm{~s}^{-1}$. The maximum amplification factor-defined here as the ratio of the wave amplitude to the amplitude of the ambient diurnal flow-is therefore about 90 .

The resonant circulation produced at $S=1.5$ is a propagating seamount-trapped wave of diurnal frequency. Brink (1989) has summarized the properties of stratified, seamount-trapped waves and discussed their dispersion properties in detail. The wavemode excited in this experiment has the gravest radial structure (mode $m=0$ ) and an azimuthal wavenumber of $n=1$. Figure 3 shows the instantaneous structure of the transport streamfunction field atop the seamount. The cyclonic and anticyclonic cells that compose the propagating wave are of comparable intensity, each carrying a transport of $0.75 \mathrm{~Sv}$. The wavemode propagates in a clockwise sense about the seamount with the period of the applied forcing.

For the mode at $S=1.5$, maximum horizontal velocities are trapped to the top and upper flanks of the seamount. Figure 4 shows the structure of the horizontal velocity field at a depth of $400 \mathrm{~m}$ (just above the seamount summit) where maximum horizontal velocities are approximately $14 \mathrm{~cm} \mathrm{~s}^{-1}$. [See also Fig. $2 \mathrm{a}$ in Brink (1989), showing the expected velocity structure for the $m=0$ mode.] Below the summit, along the flanks of the seamount, velocities are similar in pattern but weaker in amplitude (Fig. 4b). At interior levels near the top of the seamount, the perturbation density and vertical velocity fields show the azimuthal phase dependence expected for a linear propagating wave (Fig. 4a,b). In the vertical, density fluctuations are bottom trapped, with the strongest vertical velocities also occurring near the bottom (Fig. 5; cf. Brink Fig. 1a). Maximum vertical velocities are $\mathrm{O}(500-600) \mathrm{m} \mathrm{day}^{-1}$, indicating substantial deflection of the isopycnals. (It is noteworthy that even the maximum vertical velocity signal over the seamount exceeds the magnitude of the incident horizontal current.)

By contrast, the more weakly resonant mode near $S=4$, though also of azimuthal wavenumber $n=1$, has a more complicated radial structure. Alongchannel sections through the seamount at day 25 of the central experiment at $S=4.3$ are shown in Fig. 6. (The maximum resonance in the interval $4 \leqslant S \leqslant 4.5$ occurs at $S=4.3$.) The density anomaly associated with the wave (Fig. 6d) is suggestive of radial mode number 1 ( $m$ $=1$ ), and in fact the occurrence of an $m=1, n=1$ resonant mode at these parameter values will be confirmed below by comparison to the Brink (1989) dispersion results. However, the numerical wavemodes produced at $S=4.3$ have some additional vertical structure near the surface. Though we are not able to reduce our lateral viscosity to zero, this additional structure is likely an artifact of our particular choice of subgrid-scale viscosity. This is a first indication of sensitivity to the choice of viscous closure.

\section{b. Exponential stratification}

Neither the qualitative nor quantitative behavior of the resonantly generated waves appear to be a sensitive function of the detailed structure of the $N^{2}(z)$ profile. For example, a set of solutions (experiments $2 \mathrm{~A}$; Table 3 ) has been obtained using the exponential background

\section{Streamfunction at Day 25}

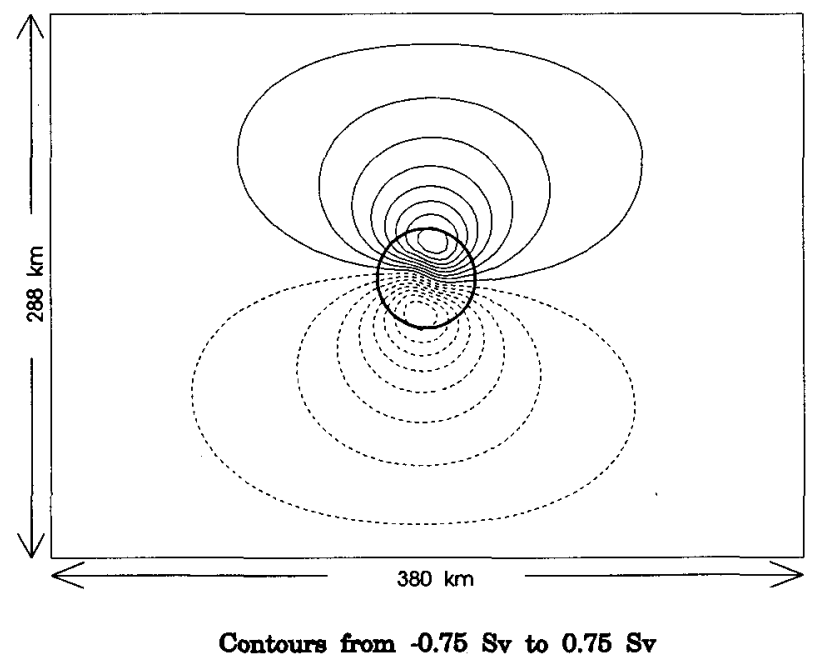

FIG. 3. Transport streamfunction at day 25 of the central experiment at $S=1.5$. The solid circle indicating size and location of the seamount has a radius of $25 \mathrm{~km}$. The contour interval (CI) equals $0.1 \mathrm{~Sv}$. (The contour lines have been symmetrically shifted about 0 ; hence, the first curves represent $\pm 0.05 \mathrm{~Sv}$.) 


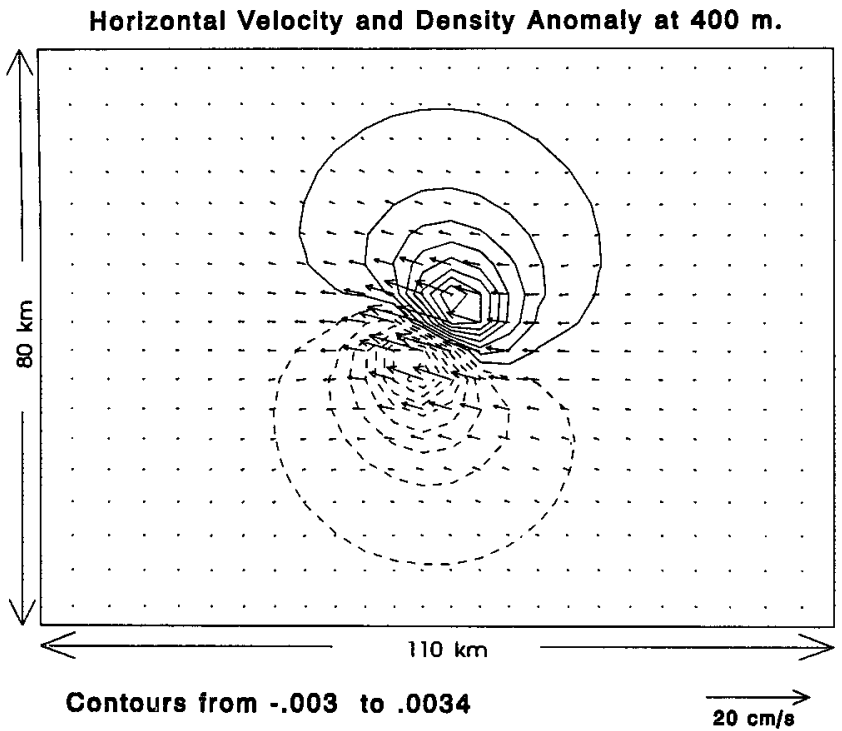

Horizontal Velocity and Vertical Velocity at $1000 \mathrm{~m}$.

FIG. 4. Horizontal velocity vectors at day 25 of the central experiment at $S=1.5$ at a depth of (a) $400 \mathrm{~m}$ [maximum vector length (MVL): $14.1 \mathrm{~cm} \mathrm{~s}^{-1}$ ] and (b) $1000 \mathrm{~m}$ [maximum vector length (MVL): $4.6 \mathrm{~cm} \mathrm{~s}^{-1}$ ] with the seamount's location and size indicated by the solid circle. Plotted on top of the vectors are (a) the density anomaly, $\mathrm{CI}=4 \times 10^{-4} \sigma_{t}$, and (b) the vertical velocity, $\mathrm{CI}=25 \mathrm{~m}$ day ${ }^{-1}$. For clarity, only the inner $110 \times 80 \mathrm{~km}$ of the horizontal domain has been plotted.
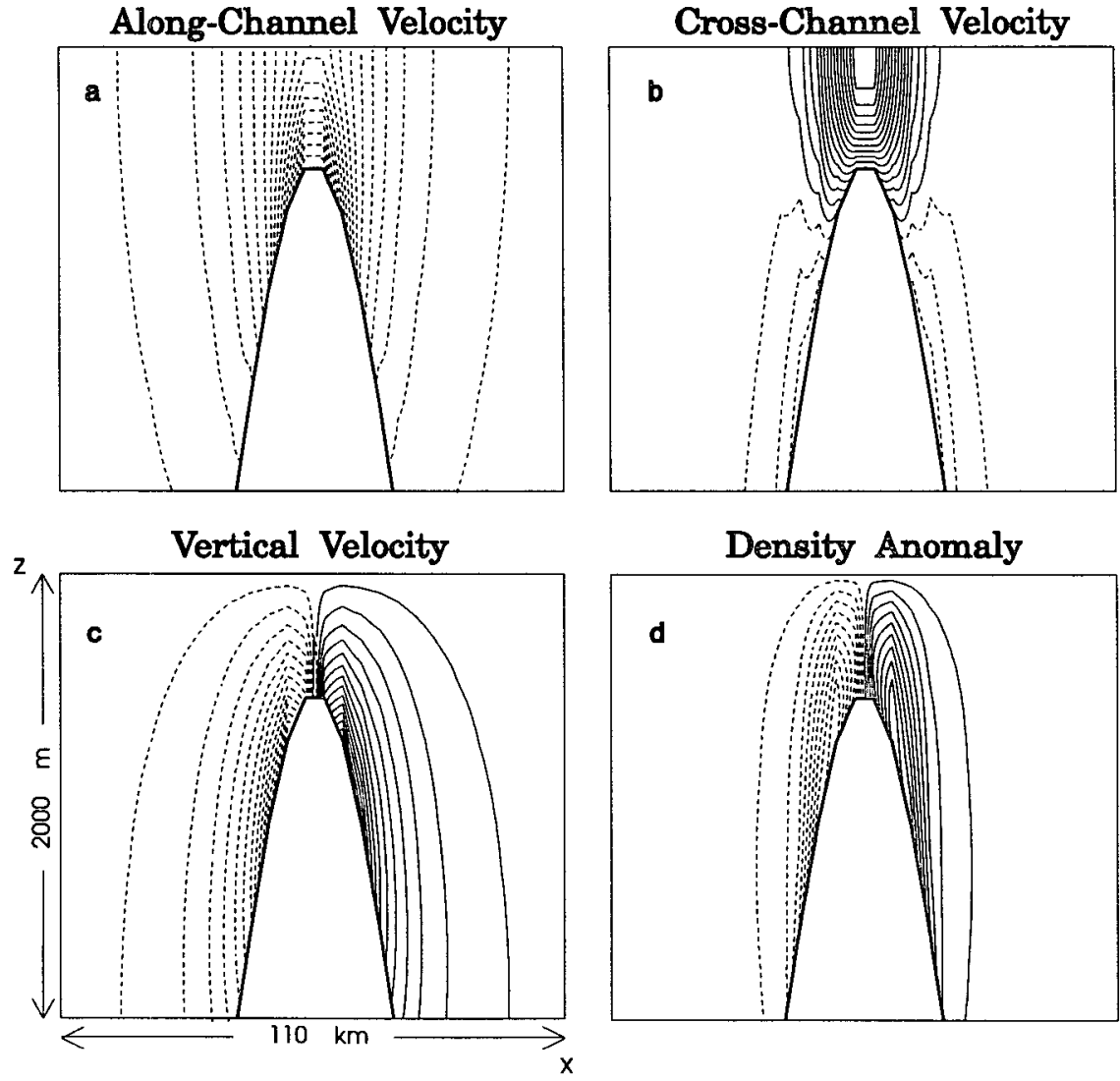

FIG. 5. Alongchannel $(x-z)$ sections through the seamount at day 25 of the central experiment at $S=1.5$ : (a) alongchannel velocity, $\mathrm{CI}=1 \mathrm{~cm} \mathrm{~s}^{-1}$; (b) cross-channel velocity, $\mathrm{CI}=0.4 \mathrm{~cm} \mathrm{~s}^{-1}$; (c) vertical velocity, $\mathrm{CI}=0.5 \mathrm{~mm} \mathrm{~s}^{-1}$; (d) density anomaly, $\mathrm{CI}=2.5 \times 10^{-4} \sigma_{t}$. For clarity, only the inner $110 \mathrm{~km}$ and the upper $2000 \mathrm{~m}$ are shown. 
Along-Channel Velocity
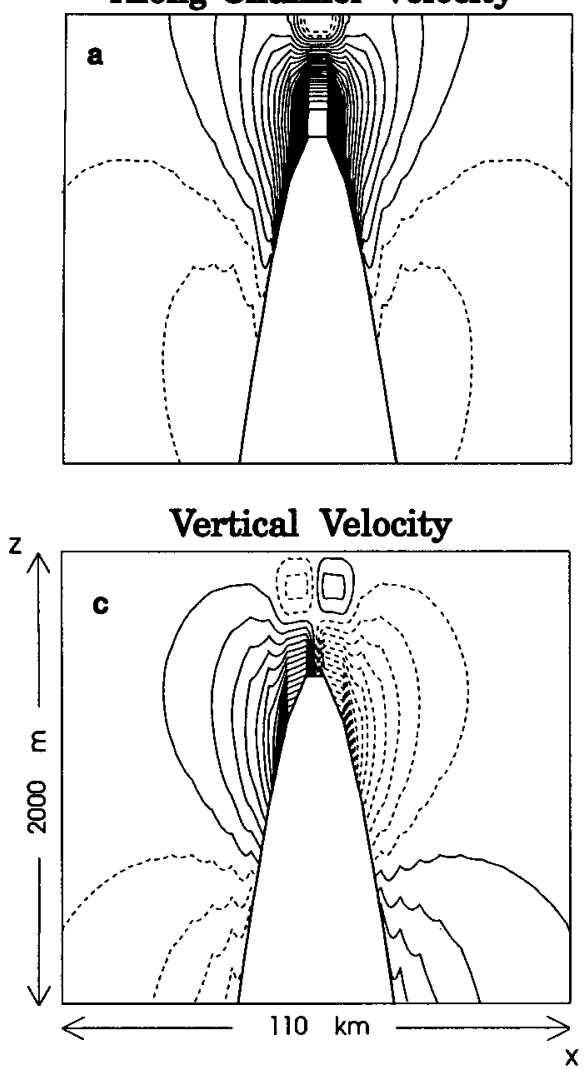

Cross-Channel Velocity

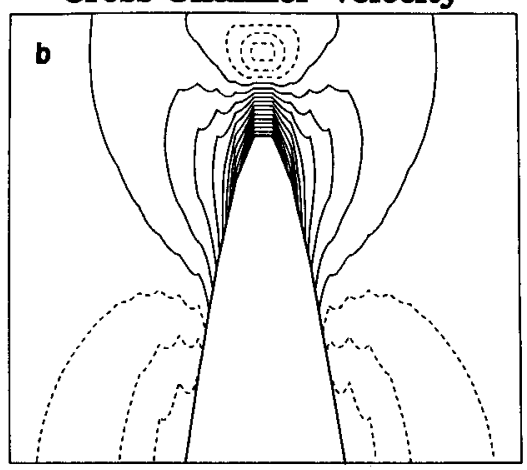

Density Anomaly

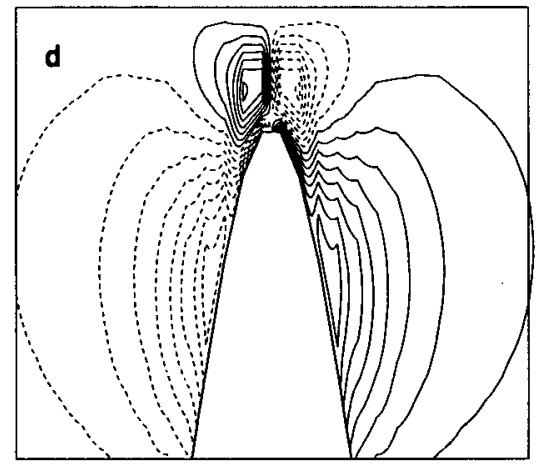

FIG. 6. Alongchannel $(x-z)$ sections through the seamount at day 25 of the central experiment at $S=4.3$ : (a) alongchannel velocity, $\mathrm{CI}=0.1 \mathrm{~cm} \mathrm{~s}^{-1}$; (b) cross-channel velocity, $\mathrm{CI}=0.2$ $\mathrm{cm} \mathrm{s}^{-1}$; (c) vertical velocity, $\mathrm{CI}=0.05 \mathrm{~mm} \mathrm{~s}^{-1}$; (d) density anomaly, $\mathrm{CI}=2.0 \times 10^{-4} \sigma_{t}$. For clarity, only the inner $110 \mathrm{~km}$ and the upper $2000 \mathrm{~m}$ are shown.

density profile, Eq. (3). The resulting dependence of wave amplitude on the bulk Burger number $S$ is seen in Fig. 7 to be comparable to that for constant stratification. In both cases, the maximally resonant circulation is the lowest mode ( $m=0, n=1)$ seamounttrapped wave. Instantaneous pictures of the density and velocity structure of the wave produced with variable $N$ (not shown) are qualitatively similar to those with constant $N$.

There are, however, several small differences between the resonance properties of the uniformly and variably stratified solutions. Perhaps most importantly, the location of the maximum response is slightly shifted from $S=1.5$ to a Burger number of approximately 1.1 . The shift of the response peak to a somewhat smaller $S$ value can be understood by recalling that for identical Burger numbers the exponentially stratified fluid will have a somewhat larger value of $N$ near the top of the seamount where the currents are most pronounced. Thus, a Burger number computed on the basis of the local value of $N$ at the seamount summit would be larger for the exponential profile. This suggests that the resonance properties of the seamount-trapped waves are responsive to the local environment at the top and upper flanks of the seamount.

Note also that the computed amplification factors for exponential stratification are somewhat smaller than those obtained for constant $N$. The maximum amplification in Fig. $7[O(70)]$ is nonetheless substantial. Lastly, although there is no reason not to expect a secondary maximum at higher Burger number to exist with exponential stratification, numerical instability of the solution to the adiabatic linearized system prevents computations beyond $S=3.5$.

\section{c. Alternate pressure gradient algorithm}

In $\mathrm{BH}$, various alternative pressure gradient algorithms were devised and tested in the search for a more accurate numerical formulation. In particular, a corrected pressure gradient algorithm (called CPG), based upon high-order vertical interpolation of the pressure field, was shown to have a dramatically reduced pressure gradient error level; unfortunately, its range of stable numerical application proved to be much more limited than the traditional gradient algorithm (OPG). 


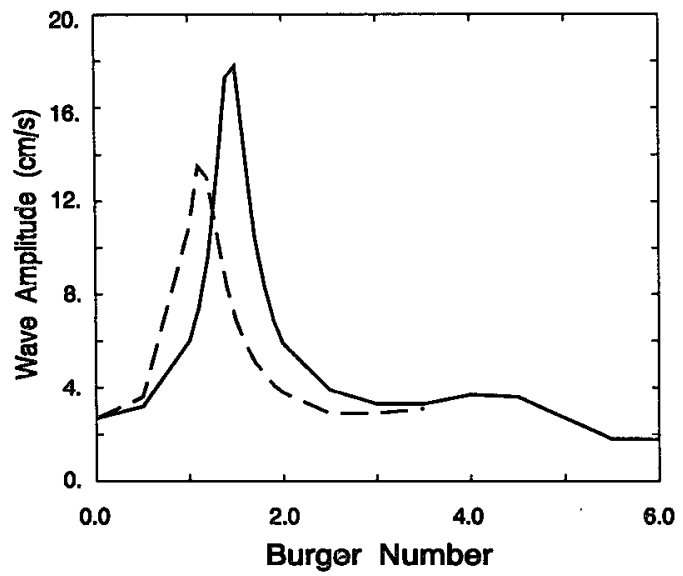

FIG. 7. Wave amplitude in centimeters per second as a function of Burger number for experiments $1 \mathrm{~B}$ (constant $N$; solid line) and 2A (exponential $N$; dashed line). The incident flow strength is 2 $\mathrm{mm} \mathrm{s}^{-1}$.

Despite the stability barrier restrictions of CPG, we are able to reproduce many of the resonance results obtained in experiments $2 \mathrm{~A}$ using OPG. Table 3 (experiments $2 B$ ) shows the results of using CPG in the case of exponential stratification. As expected, the use of $\mathrm{CPG}$ is restricted to a narrower range of Burger number $(S<1.5)$. Nonetheless, this range is large enough to span the resonance peak at $S=1.1$. The amplification factors produced by the two pressure gradient treatments are virtually identical near resonance. This is additional assurance that the amplified waves seen here are not numerical artifacts.

\section{d. Dependence on forcing frequency}

Motivated by the observed enhancement of diurnal currents at the locations mentioned in the Introduction, we have thus far held the frequency of the applied forcing fixed at the diurnal frequency. Substantial resonances are possible at other forcing frequencies as well, however, so long as there is a "close enough" match between the forcing frequency and the frequency of an admissible free wave. Exactly how close is close enough is of course one of the important issues, in that the breadth of the resonance phenomenon will dictate in part the likelihood of its observance in nature.

Figure 8 summarizes the results of a set of solutions in which forcing frequency and Burger number were simultaneously varied. The results confirm the existence of significant amplification of currents over the seamount for a wide range of subinertial frequencies and Burger numbers. As expected, the maximum amplification occurs for $S / \omega$ combinations corresponding to freely propagating waves. The amplification factor decreases rapidly as it approaches the superinertial regime in which no free waves are possible.

\section{e. The effects of dissipation}

It is to be expected in this resonantly forced system that the specific choice of dissipation will matter a great deal. In particular, it is dissipation that limits the amplification of the free wave. This is an unfortunate circumstance, since the "proper" parameterization of subgrid-scale mixing of momentum and density in numerical ocean circulation models is highly uncertain. Our prejudice here has been to use as little explicit smoothing as necessary to obtain stable solutions, and where smoothing is required to use "traditional" operator forms. In the linearized solutions, these forms include a biharmonic viscosity formulation applied along sigma surfaces, a linear bottom drag applied as a body force at $z=-h$, and a depth-independent Rayleigh damping on the horizontal velocity components $u$ and $v$. [Scale-selective lateral mixing is now a common choice in large-scale numerical ocean modeling; bottom friction and/or Rayleigh damping have been more traditionally employed in theoretical studiesfor example, Wright and Loder (1985) and Maas and Zimmerman (1989a,b).]

In the presence of biharmonic lateral smoothing alone, relatively modest changes in the coefficient of biharmonic viscosity have a proportional effect on the strength of the resonance mechanism. For example, an increase (decrease) of the coefficient by a factor of $\sqrt{10}$ produces a roughly equivalent decrease (increase) in the amplitude of the resonance peak (Fig. 9; Table 3 ). Uncertainty in $\nu$ of an order of magnitude is thus associated with a comparable variation in the maximum amplification of the free wave. Given the wide range of values for $\nu$ currently used in ocean circulation modeling, not to mention the associated issue of the choice of operator form, this is a substantial sensitivity indeed.

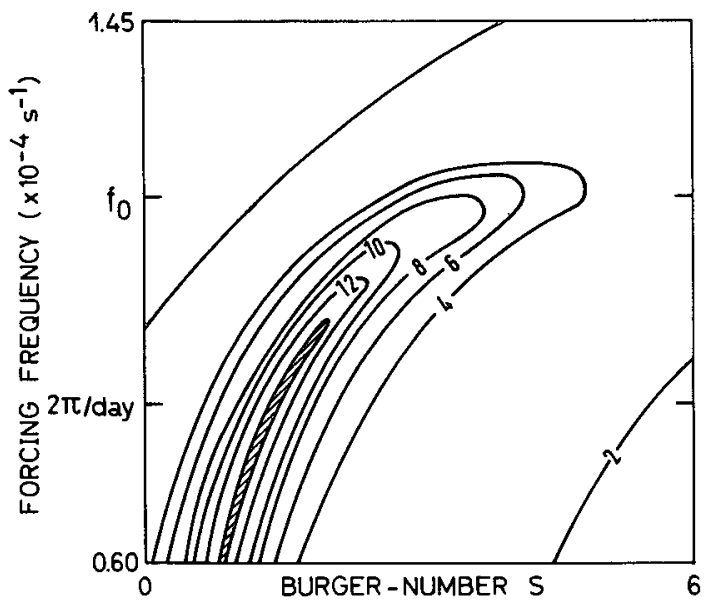

FIG. 8. Wave amplitude in centimeters per second as a function of Burger number and forcing frequency for the linearized equations. The incident flow strength is $2 \mathrm{~mm} \mathrm{~s}^{-1}$. Values greater than $14 \mathrm{~cm} \mathrm{~s}^{-1}$ are shaded. 


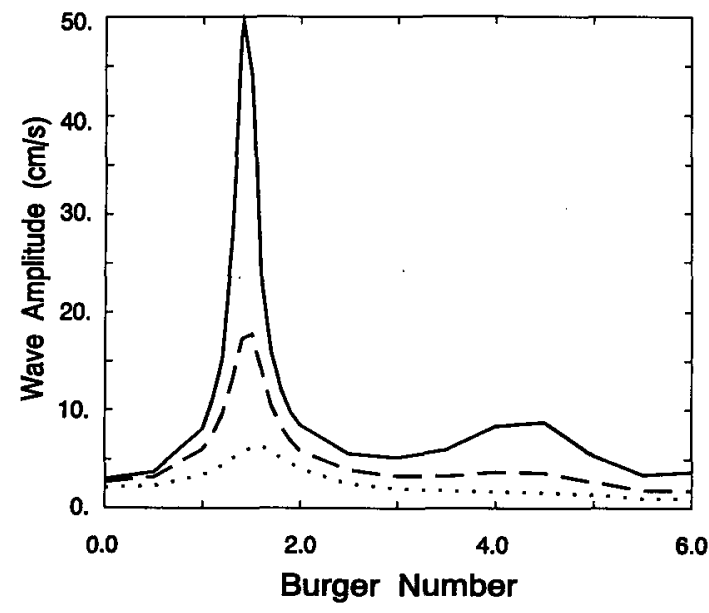

FIG. 9. Wave amplitude in centimeters per second as a function of Burger number for experiments 1B (dashed curve), 1C (dotted curve; increased viscosity), and $1 \mathrm{~A}$ (solid curve; decreased viscosity). The incident flow strength is $2 \mathrm{~mm} \mathrm{~s}^{-1}$.

Some scale-selective viscosity is necessary for the stable execution of these resonance simulations. Nonetheless, if either bottom friction or Rayleigh damping are added, the coefficient of biharmonic viscosity can be reduced substantially, to the point where lateral mixing is secondary to the momentum balance. (This is quantified below when we consider the timemean dynamic balances.)

Figure 10 summarizes the amplification factors obtained for two sequences of experiments, one employing a combination of bottom and lateral friction, and a second using Rayleigh damping and lateral friction. In the former, the dimensional bottom drag coefficient is $r_{d}=2.6 \times 10^{-3} \mathrm{~m} \mathrm{~s}^{-1}$; in the latter, $r_{\mathrm{Ra}}=1 /(2$ days). The resulting nondimensional viscous parameters are shown in Table 1. The important point made by Fig. 10 is that the resonant waves produced here can be equilibrated equally well by the various combinations of viscous processes that we have tried. In all cases, the qualitative properties of the trapped waves are similar. Maximum amplification factors range between 40 and 120 for these choices of subgrid-scale parameters.

\section{f. Comparison with prior analytic studies}

The excitation of subinertial free waves about isolated topographic features has previously been studied in the linearized limit by both Chapman (1989) and Brink (1990). The former work considered a simple model of an oscillating, rectilinear, barotropic current flowing over a radially symmetric topographic bump and damped by bottom friction. The latter model included continuous density stratification, and considered the case of horizontally and vertically sheared incident flow. The model adopted here is dynamically analogous to these, except for the inclusion of lateral subgrid-scale mixing of momentum. (Also, we focus exclusively on barotropic forcing.)

Various quantitative and semiquantitative comparisons with these prior studies are possible. The most unambiguous of these is a comparison of the location of the resonance peak with the location implied by the dispersion relation for freely propagating seamounttrapped waves. Brink (1989) has discussed the effects of stratification on the propagation of seamounttrapped waves, and computed the dispersion properties of the waves for a wide range of Burger numbers. We have duplicated his dispersion calculations here, using his original code, to verify that the numerically determined wave resonance lies on or near the appropriate dispersion curve.

The results of these dispersion calculations indicate for linear stratification that the maximum resonance should occur at a Burger number of $S=1.45 \pm 0.01$. The numerical solutions (experiment $1 \mathrm{~B}$; Table 3 ) indicate a resonance peak somewhere between the Burger number values of 1.4 and 1.5 , though perhaps somewhat closer to the latter value. Nonetheless, the agreement with the dispersion estimates is quite good. The analogous comparison for an exponential background stratification is also favorable. The solutions obtained here suggest that the resonance occurs between $S$ $=1.1$ and 1.2 ; the computed dispersion properties of the waves place the resonance at an $S$ value of 1.17 \pm 0.01 . In both cases, the resonant modes are identified to have wavenumbers $m=0$ and $n=1$. Lastly, a secondary resonance is found with linear stratification to occur at $S=4.32 \pm 0.01$, also in agreement with the numerical results; the structure of this latter mode is confirmed to be $m=1$ and $n=1$. The existence of only two resonant modes ( $m=0$ and $m=1)$ within our range of $S$ is consistent with Brink's (1989) analysis,

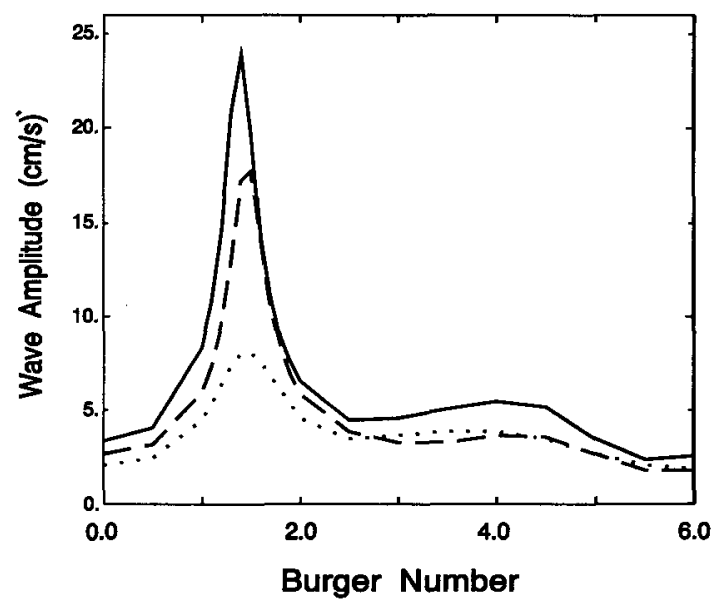

FIG. 10. Wave amplitude in centimeters per second as a function of Burger number for experiments 1B (dashed curve; lateral viscosity), ID (solid curve; bottom friction), and IE (dotted curve; Rayleigh damping). The incident flow strength is $2 \mathrm{~mm} \mathrm{~s}^{-1}$. 
which shows that the higher radial modes occur only at much higher Burger numbers for a diurnal forcing (e.g., see his Fig. 4).

The magnitude of the amplification experienced by the propagating wave is another important measure of the free wave excitation mechanism. Although precise quantitative comparisons are not possible, the maximum velocity amplification factors produced near resonance in the numerical solution $[O(100)]$ are consistent with those computed by Brink (1990). (In Brink's study, the primary amplification measure was kinetic energy. The KE of the free trapped waves near resonance was found to be $10^{4}$ to $10^{5}$ times greater than that of the incident flow. This corresponds to a velocity amplification of 100 to 300 .) The maximum amplification factor obtained in the central experiments are somewhat smaller than the largest factors produced by Brink's analysis; however, they are highly sensitive to the assumed values of our viscous coefficients.

All studies show a strong enhancement of the excitation as frictional parameters-the bottom resistance coefficient in the Chapman and Brink studies, and the biharmonic viscosity coefficient here-are reduced in magnitude. Our results are also consistent with these prior studies in suggesting that for horizontally uniform incident flow the lowest-mode seamount-trapped waves will be excited preferentially over the higher modes (Brink 1990). This appears to be true despite the rather different dissipative assumptions adopted in the respective studies.

\section{The effects of nonlinearity}

Next, we consider the effects of retaining nonlinear advection of density and momentum in the governing equations. In the presence of nonlinearity, additional processes are possible. Among these is the generation of significant residual (time mean) circulation about the seamount. The occurrence of permanent circulation in the neighborhood of the seamount is a matter of considerable concern. For example, such systematic circulation patterns are clearly consequential to the local redistribution of chemical and biological properties. We investigate the issue of residual circulation next in the context of the fully nonlinear system of equations $(5)-(10)$.

\section{a. Preliminary considerations}

It is obvious that the linearized versions of the continuum equations, forced in a purely oscillatory manner, should yield a purely oscillatory response having no pointwise residual circulation. Unfortunately, this result is not true of the discretized version of the equations of motion, which may (depending on the approximation procedures utilized) produce a nontrivial mean flow even in the linearized limit. A necessary condition for avoiding spurious time-mean flows is that an initially resting ocean with level isopycnal surfaces should be a stationary solution of the discretized equations. Spurious accelerations can arise for several reasons. A first has already been mentioned: the sigma coordinate system is known to be associated with systematic errors in its representation of the pressure gradient forces. As discussed by $\mathrm{BH}$, these errors may accumulate to produce mean flows of nontrivial magnitude. Fortunately, for the numerical parameter settings used here, $\mathrm{BH}$ show that the mean flow errors are quite small, being of order $10^{-6} \mathrm{~m} \mathrm{~s}^{-1}$.

Errors in the pressure gradient estimates are not the only means of generating unanticipated residual currents, however. Particular forms for subgrid-scale diffusion may also have this property. In particular, addition to the density equation of diffusion oriented along sigma surfaces will produce a mean anticyclonic circulation about the seamount even in the absence of forcing. The circulation arises because diffusion along sigma surfaces is equivalent to a spatially variable vertical mixing of the density field, which acts to produce upwardly tilted isopycnals at the seamount. The resulting geostrophically balanced mean flow need not be insignificant. For example, if we add a small amount $\left(1 \times 10^{8} \mathrm{~m}^{4} \mathrm{~s}^{-1}\right.$ ) of biharmonic diffusion to the central experiment at $S=1.5$ (experiment $1 \mathrm{~B}$ ), the maximum wave amplitude is virtually unchanged but a mean flow of $O(1) \mathrm{cm} \mathrm{s}^{-1}$ is produced. This effect-which if not spurious, is undesirable in these calculations-can be avoided by diffusing the perturbation density only, that is by diffusing the difference between the instantaneous density field and the resting stratification $\rho_{r}(z)$. This ensures that a resting initial state with $\rho=\rho_{r}$ is a stationary solution of the equations, and rules out the occurrence of appreciable time-mean flow as an artifact of the subgrid-scale diffusion operator.

TABLE 4. Wave mean amplitudes $\left(\mathrm{cm} \mathrm{s}^{-1}, \mathrm{~cm} \mathrm{~s}^{-1}\right)$ for experiments with nonlinear physics, forced with $2 \mathrm{~mm} \mathrm{~s}^{-1}$ at 1 -day period. An asterisk denotes a numerically unstable experiment.

\begin{tabular}{lccr} 
& \multicolumn{3}{c}{ Experiment } \\
\cline { 2 - 4 }$S$ & $3 \mathrm{~A}$ & $3 \mathrm{~B}$ & \multicolumn{1}{c}{$3 \mathrm{C}$} \\
\hline 0.0 & $2.7,0.0295$ & $2.7,0.0295$ & $2.2,0.0191$ \\
0.2 & $2.7,0.0312$ & $2.7,0.0313$ & $2.3,0.0208$ \\
0.4 & $3.0,0.0381$ & $3.0,0.0382$ & $2.5,0.0270$ \\
0.6 & $3.5,0.0554$ & $3.5,0.0555$ & $3.1,0.0493$ \\
0.8 & $4.4,0.0978$ & $4.3,0.0982$ & $4.0,0.1243$ \\
1.0 & $6.0,0.2192$ & $6.0,0.2199$ & $5.9,0.3865$ \\
1.1 & $7.5,0.3681$ & $7.4,0.3687$ & $7.3,0.6738$ \\
1.2 & $9.7,0.6823$ & $9.6,0.6730$ & $9.0,1.1785$ \\
1.3 & $*$ & $13.0,1.3383$ & $11.2,2.2413$ \\
1.4 & $*$ & $17.7,2.6261$ & $13.8,4.2449$ \\
1.5 & $*$ & $19.7,3.3795$ & $16.1,5.8768$ \\
1.6 & $*$ & $15.5,2.3154$ & $13.4,4.9474$ \\
1.7 & $*$ & $11.3,1.3177$ & $10.8,3.7593$ \\
1.8 & $*$ & $8.8,0.8446$ & $9.3,2.9931$ \\
1.9 & $7.9,0.7724$ & $7.3,0.6015$ & $8.3,2.4883$ \\
2.0 & $6.5,0.5263$ & $6.2,0.4637$ & $7.7,2.1339$ \\
\hline & & &
\end{tabular}




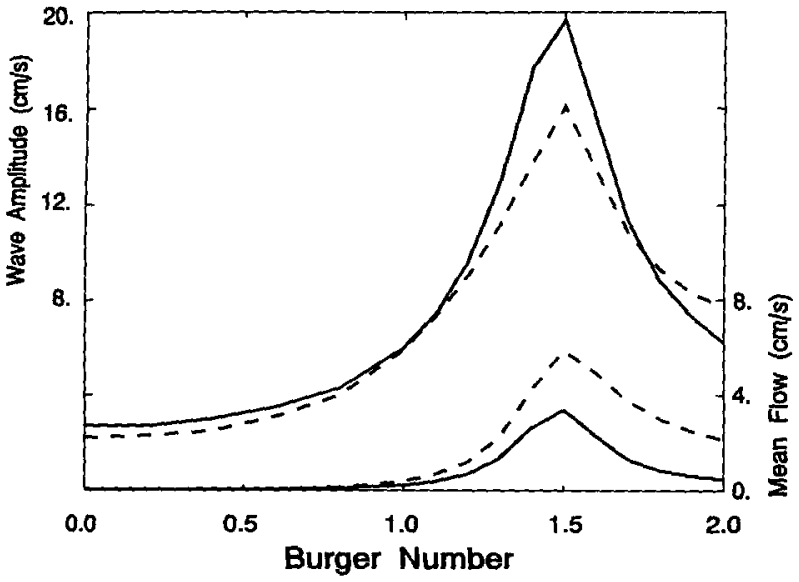

FIG. 11. Wave amplitude and mean flow strength in centimeters per second for the nonlinear experiments $3 \mathbf{B}$ (solid curves; lateral viscosity) and $3 \mathrm{C}$ (dashed curves; Rayleigh damping). The incident flow strength is $2 \mathrm{~mm} \mathrm{~s}^{-1}$.

\section{b. Parameter dependence of the mean flow amplitude}

Table 4 summarizes three of the sequences of nonlinear solutions we have obtained. As in the linearized experiments, solutions were determined for a wide range of Burger number, and for a variety of subgrid- scale viscous closures. A small amount of biharmonic diffusion $\left(\nu_{\rho}=1 \times 10^{8} \mathrm{~m}^{4} \mathrm{~s}^{-1}\right)$ acting on the perturbation density field was required in the nonlinear runs to produce numerically stable integrations across the full range of Burger number $0<S<2$. However, where duplicate experiments could be carried out both with and without density diffusion, the differences in wave and mean flow amplitudes were observed to be small (cf. Table 4, experiments $3 \mathrm{~A}$ and $3 \mathrm{~B}$ ). As above, comparable results are obtained for both linear and exponential density profiles. We will explicitly discuss only the constant $N^{2}$ case here.

The nonlinear solutions confirm that appreciable time-mean currents can be generated in the neighborhood of the seamount. The mean flows increase rapidly in magnitude as resonance is approached, reaching peak values as great as $37 \%$ of the free-wave amplitude. Figure 11 shows both the wave and mean flow amplitudes as a function of Burger number for experiments $3 \mathrm{~B}$ and $3 \mathrm{C}$. Experiments $3 \mathrm{~B}$ are identical to the central set of experiments $1 \mathrm{~B}$ except for the aforementioned addition of nonlinearity and a weak diffusivity. Experiments $3 \mathrm{C}$ have a greatly reduced (by a factor of 100 ) value of lateral viscosity plus a nonzero Rayleigh damping term. (Table 1 summarizes the parameter values.)

\section{Instantaneous Streamfunction}

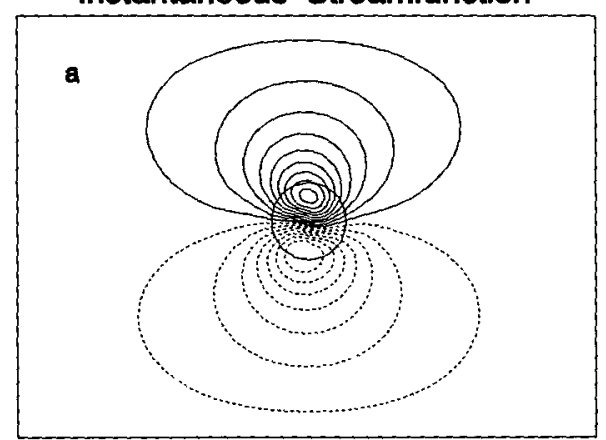

Time-Mean Streamfunction

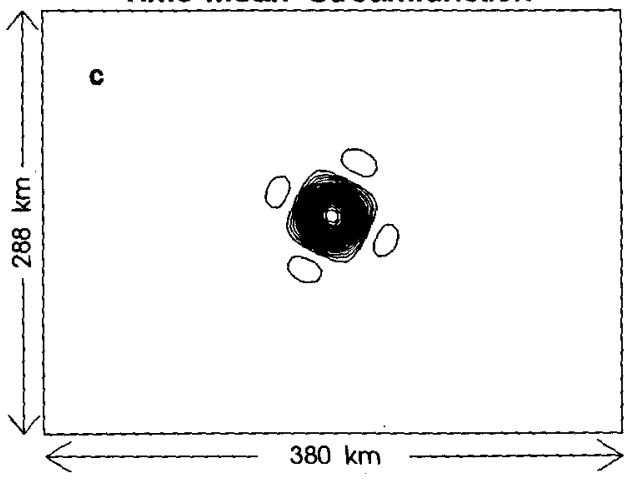

Diurnal Streamfunction

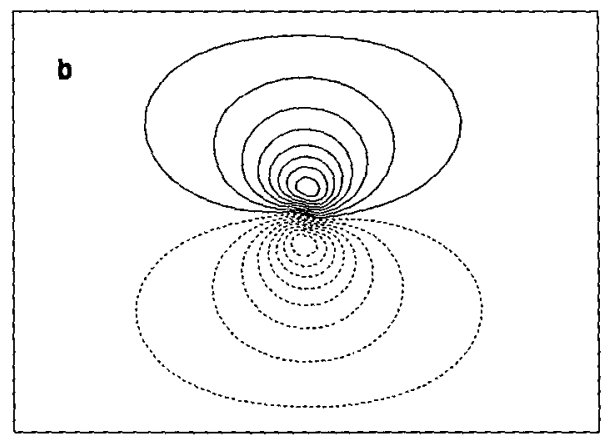

Semi-Diumal Streamfunction

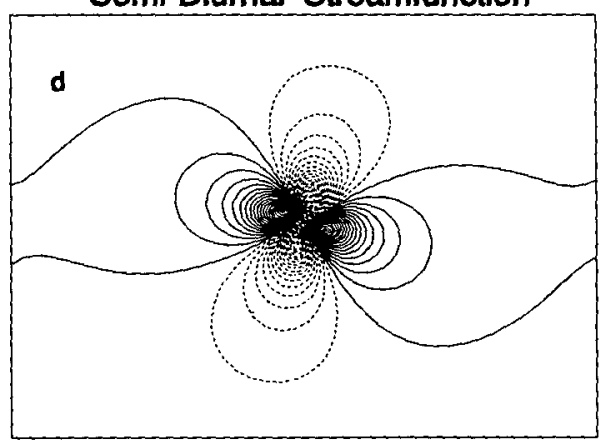

FIG. 12. Spectral decomposition of the transport streamfunction of the nonlinear experiment $3 \mathrm{~B}$ at $S=1.5$. (a) Instantaneous; $\mathrm{Cl}=0.1 \mathrm{~Sv}$, (b) diurnal period; $\mathrm{Cl}=0.1 \mathrm{~Sv}$, and (c) time mean; $\mathrm{Cl}=0.01 \mathrm{~Sv}$, (d) semidiurnal period; $\mathrm{Cl}=0.001 \mathrm{~Sv}$. All other frequency components are significantly smaller. 
The inclusion of advection of momentum and perturbation density produces a slight increase in the instantaneous amplitude of the currents atop the seamount. Compare, for example, experiments $1 \mathrm{~B}$ and 3 B at $S=1.5$, which produce wave amplitudes of 17.8 and $19.7 \mathrm{~cm} \mathrm{~s}^{-1}$, respectively. The location of the maximum resonance $(S=1.5)$, and the breadth of the resonance peaks are also largely unaffected. Significant residual circulation has, however, been generated; maximum mean flows are found to be 3.4 $\mathrm{cm} \mathrm{s}^{-1}$ and $5.9 \mathrm{~cm} \mathrm{~s}^{-1}$ for experiments $3 \mathrm{~B}$ and $3 \mathrm{C}$. This represents a "rectification efficiency"- the ratio of maximum mean flow to wave amplitude-of $17 \%$ and $37 \%$, respectively.

\section{c. Frequency decomposition of the instantaneous circulation}

The instantaneous transport streamfunction associated with the propagating nonlinear wavemode is shown in Fig. 12a. It is qualitatively similar in amplitude and structure to the results of the linearized model (cf. Fig. 3); however, whereas the linearized wavemode was composed of cyclonic and anticyclonic cells of approximately equal amplitude, the nonlinear waveform is now asymmetric. The cyclonic cell has been reduced in transport amplitude by roughly $15 \%$, and the anticyclonic circulation augmented by a similar amount. Nor is the external (depth integrated) flow field the only feature to be rendered asymmetric by advection. Vertical slices taken through the seamount (Fig. 13) show significant asymmetry in all fields. (Compare with the linearized results in Fig. 5.) Note the strengthening of the downwelling circulation, and the appearance of a largely positive density anomaly over the seamount.

The time-evolving structure of the circulation may be better understood by performing a frequency-space analysis of a time series of instantaneous streamfunction fields. We have done so by analyzing a time series of $128 \psi$ fields uniformly spaced in time over days 25 to 29 of experiment 3B $(S=1.5)$. The amplitude and spatial structure of the three dominant frequency components are shown in Figs. 12b-d.

Overall, the diurnal frequency component is dominant, and largely unchanged in either structure or amplitude from the patterns arising in the linearized ex-
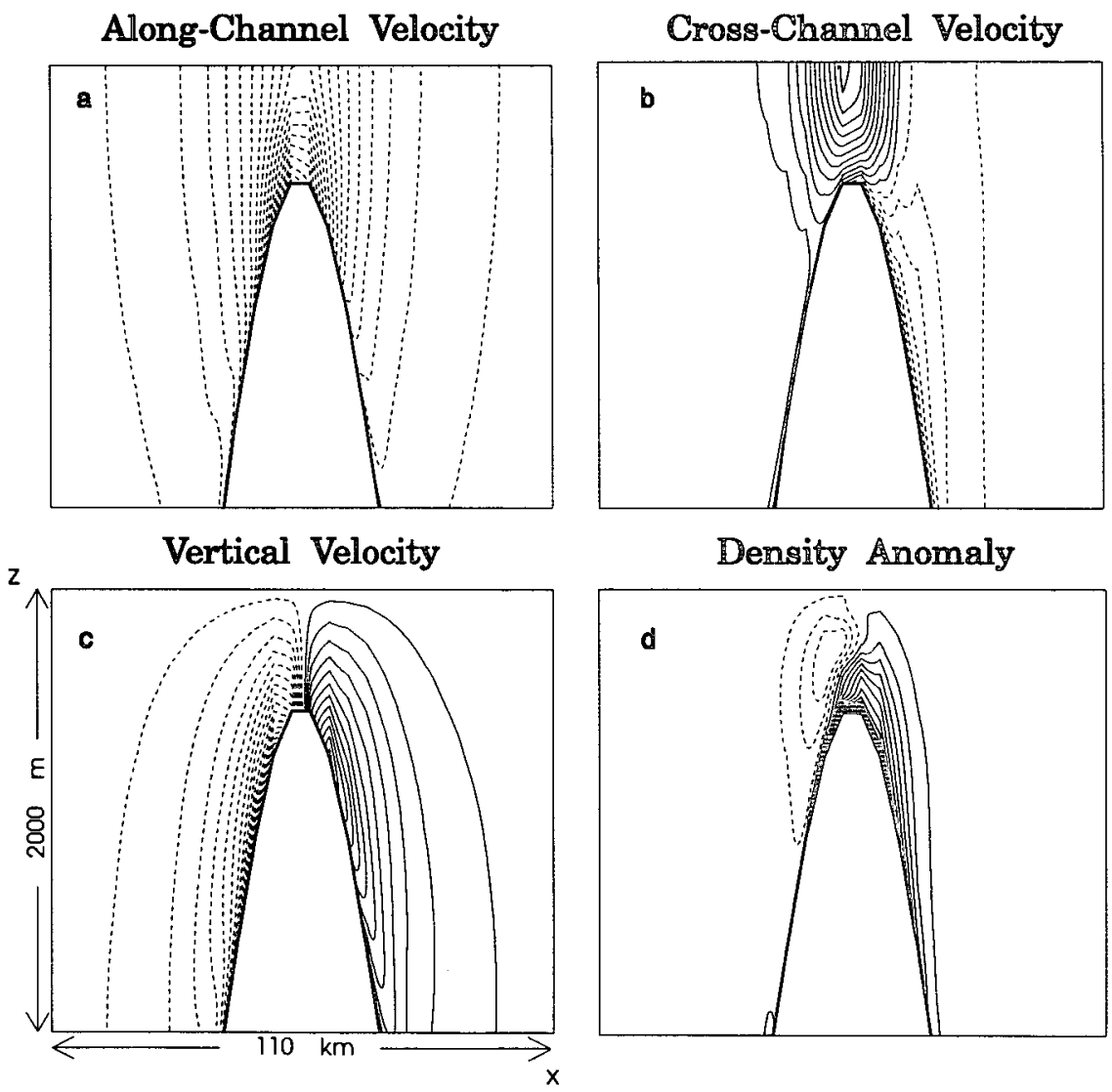

FIG. 13. Alongchannel $(x-z)$ sections through the seamount at day 25 of the nonlinear experiment $3 \mathrm{~B}$ at $\mathrm{S}=1.5$ : (a) alongchannel velocity, $\mathrm{CI}=1 \mathrm{~cm} \mathrm{~s}^{-1}$; (b) cross-channel velocity, $\mathrm{CI}$ $=0.5 \mathrm{~cm} \mathrm{~s}^{-1}$; (c) vertical velocity, $\mathrm{CI}=0.5 \mathrm{~mm} \mathrm{~s}^{-1}$; (d) density anomaly, $\mathrm{CI}=5 \times 10^{-4} \sigma_{t}$. For clarity, only the inner $110 \mathrm{~km}$ and the upper $2000 \mathrm{~m}$ are shown. 
periment (cf. Fig. 12b with Fig. 3). The zero frequency circulation is next most intense, and corresponds to an azimuthally invariant high pressure cell (a net anticyclonic circulation; Fig. $12 \mathrm{c}$ ) atop the seamount. The diurnal and time-mean frequency components together account for the major features of the instantaneous circulation-that is, it is the residual circulation that causes the asymmetry of the instantaneous wave. The next most intense frequency component, the semidiurnal, has a quadrupole structure (azimuthal mode number $n=2$, Fig. 12d) but is weaker by an order of magnitude than the time-mean circulation.

\section{d. The residual circulation: Kinematic structure}

The time-mean transport streamfunction (Fig. 12c) is dominated by a single anticyclonic circulation. The lateral scale of the residual barotropic circulation is narrow, comparable to that of the seamount itself. The mean transport for the central parameters is roughly $0.25 \mathrm{~Sv}$. A set of four weak ancillary anticyclonic cells surrounds the inner region of anticyclonic flow. It is not certain whether these weaker circulation features are inherent in the continuum problem (at least, we see no way to rule them out) or a consequence of the Cartesian grid used to obtain the numerical solution.

Vertical sections through the center of the seamount (Fig. 14) indicate that the time-mean horizontal circulation is bottom intensified, with the strongest flows occurring along the flanks of the seamount at depths of 800 to $1000 \mathrm{~m}$. Maximum residual flows exceed 3 $\mathrm{cm} \mathrm{s}^{-1}$ at these levels but are small just above the seamount summit at $400 \mathrm{~m}$. This is in contrast with the structure of the free wave, which was enhanced near the top of the seamount relative to deeper levels (Fig. 5).

Although the primary circulation is directed in a clockwise sense about the seamount, a strong secondary circulation exists in the vertical-radial plane as well. In particular, on average there is downwelling over the top and upper flanks of the seamount, with weaker upwelling somewhat farther out (Fig. 14c). The convergence of fluid associated with this systematic vertical downwelling is balanced by net radial export of fluid near the top of the seamount and by a weaker import near the surface (Fig. 14a). Isopycnals are depressed in the upper water column by the persistent downwelling (Fig. 14d); however, they are upwardly tilted
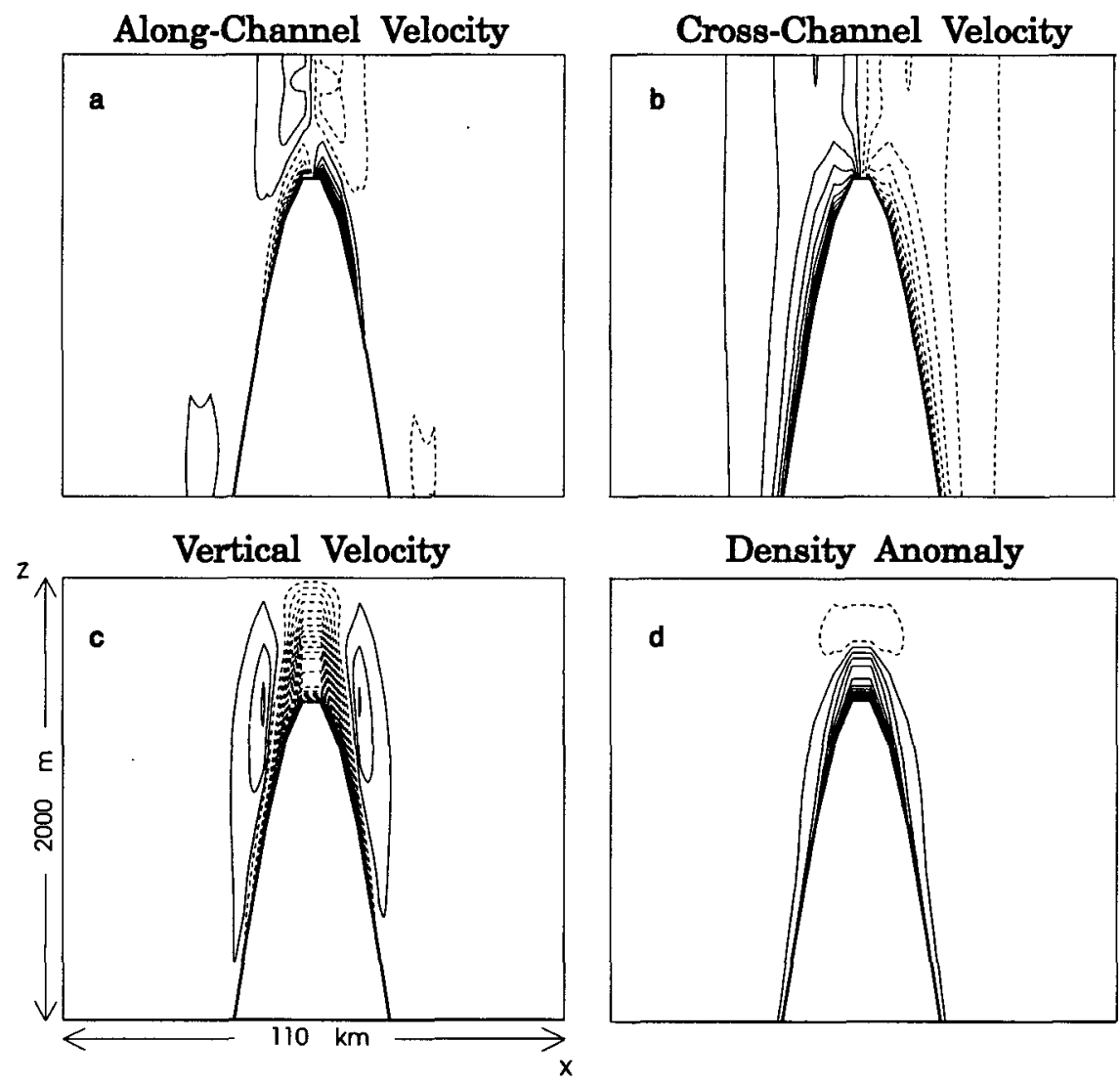

FIG. 14. Time-mean alongchannel $(x-z)$ sections through the seamount of the nonlinear experiment 3B at $S=1.5$ : (a) mean alongchannel velocity, $\mathrm{CI}=0.1 \mathrm{~cm} \mathrm{~s}^{-1}$; (b) mean cross-channel velocity, $\mathrm{CI}=0.25 \mathrm{~cm} \mathrm{~s}^{-1}$; (c) mean vertical velocity, $\mathrm{Cl}=2 \mathrm{~m}$ day $^{-1}$;(d) mean density anomaly, $\mathrm{CI}=5 \times 10^{-4} \sigma_{t}$. For clarity, only the inner $110 \mathrm{~km}$ and the upper $2000 \mathrm{~m}$ are shown. 
near the seamount itself, as required by local geostrophy of the strong azimuthal flow. The positive density anomaly, though geostrophically consistent with the anticyclonic residual flow, might seem at odds with the net downwelling over the seamount. The positive anomaly is maintained against vertical advection of lighter fluid by the radial flow, which acts to restore the positive anomaly.

\section{e. The residual circulation: Dynamical interpretation}

To explore the dynamical basis for the residual circulation we have computed the time-mean momentum balances that give rise to the strong residual anticyclonic flow about the seamount. Given the geometry of the seamount and the inherent interest in the production of rotary mean currents, it is most natural to examine the mean momentum balances in terms of their radial and azimuthal components, rather than in their Cartesian $(x$ and $y$ ) form. Although the numerical model has utilized the latter in its solution procedure, we are nonetheless able to produce an approximate set of ra- dial and azimuthal time-mean momentum balances in the following way.

Written in vector form, and after time averaging, the Cartesian equations of motion can be written as

$$
0=-\overline{h f \mathrm{k} \times \mathrm{v}}-\overline{h \nabla \phi}-\overline{(h \mathrm{v} \cdot \nabla) \mathrm{v}}+\overline{D_{\mathrm{v}}},
$$

which merely requires a balance between the vector sum of all Coriolis, pressure gradient, advective, and viscous forces. To produce azimuthally averaged balances appropriate to the radial and azimuthal velocity components, respectively, we evaluate an approximation to the following azimuthal integral:

$$
\begin{aligned}
\frac{1}{2 \pi r_{o}} \oint_{r=r_{o}} \hat{\lambda} \cdot[-\overline{h f \mathrm{k} \times \mathbf{v}} & -\overline{h \nabla \phi} \\
& \left.-\overline{(h \mathbf{v} \cdot \nabla) \mathbf{v}}+\overline{D_{\mathbf{v}}}\right] d \theta,
\end{aligned}
$$

where $\hat{\lambda}$ is either the unit vector in the azimuthal (clockwise) or radial (outward) direction. The approximation used here is expedient, but accurate enough for the balances presented below. We have approximated the azimuthal integral by a sum over four
Coriolis

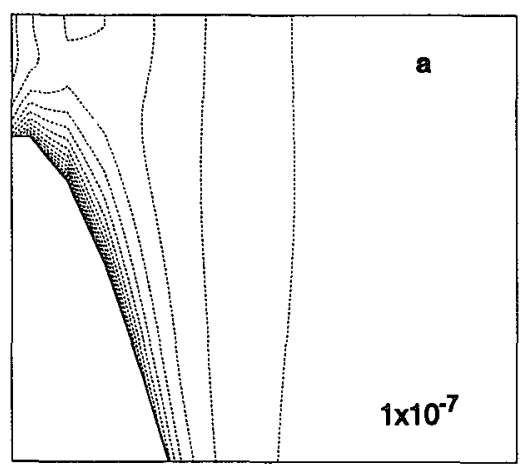

Advection

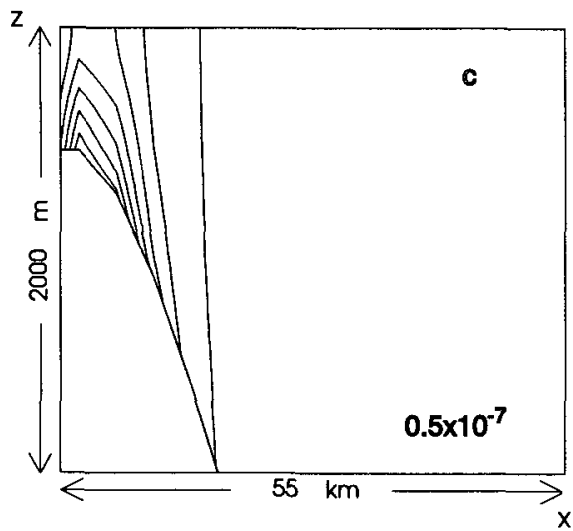

Pressure Gradient

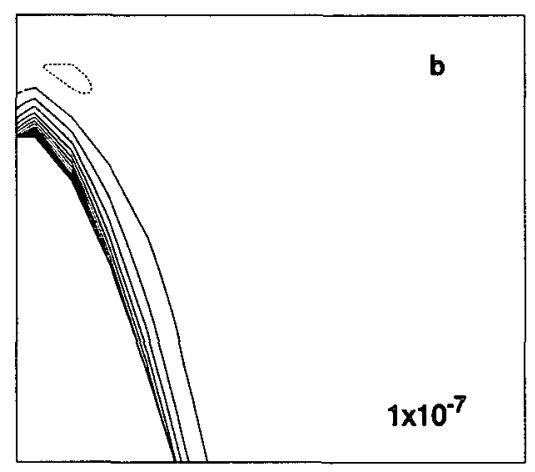

Lateral Viscosity

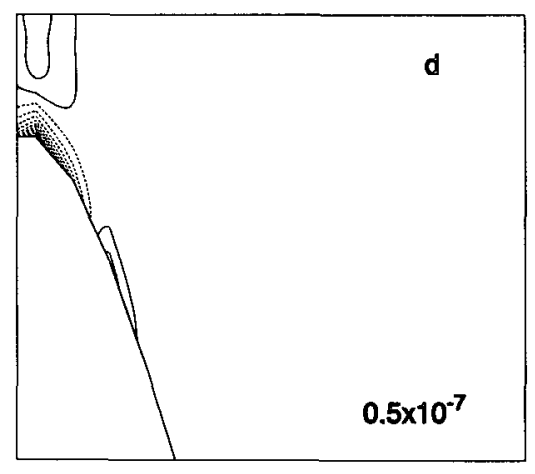

FIG. 15. Time-mean and azimuthally averaged momentum balance terms for the radial component of velocity as a function of depth $(-2000 \mathrm{~m} \leqslant z \leqslant 0)$ and radial distance $(r \leqslant 55 \mathrm{~km})$ from the seamount summit from experiment 3B at $S=1.5$ : (a) Coriolis force, (b) pressure gradient force, (c) total advective terms ( mean plus eddy; horizontal plus vertical), and (d) lateral diffusion. $\mathrm{CI}$ as noted on the panels. 
radial sections on the two cross-channel and two alongchannel flanks of the seamount. This eliminates the need for any interpolation between model grid points, which itself might be a source of approximation error.

The resulting time-mean radial and azimuthal momentum balances for experiment 3B $(S=1.5)$ are shown in Figs. 15 and 16, respectively. The time-mean radial momentum balance is geostrophic to leading order, with both the Coriolis and pressure gradient forces (Fig. 15a,b) showing the bottom-intensified pattern of the time-mean azimuthal velocity component. Despite the tendency toward geostrophy, however, the geostrophic residual (the difference between the Coriolis and pressure gradient terms) is not small, being roughly $15 \%-20 \%$ in amplitude as large as either of the dominant terms. The deficit in the time-mean radial balance is made up mutually by total (mean plus eddy) advection of momentum and by lateral viscous effects. The former, entirely dominated by lateral eddy transport of momentum, acts to systematically transfer positive (outward) radial momentum from the far field to the upper flanks of the seamount (Fig. 15c). The latter acts to redistribute the radial momentum from the upper seamount to slightly deeper levels (Fig. 15d). The sum of the four time-mean momentum balance terms is small, $\mathrm{O}(0.1 \%)$ of the largest individual terms, showing that the time-averaging interval is sufficient for accurate recovery of the mean dynamical balances.

The azimuthally averaged balances in the azimuthal direction cannot be geostrophic at leading order. In the limit of perfect resolution, the azimuthal integral of the pressure gradient force in the azimuthal momentum equation must vanish exactly since it represents the integral around a closed loop of a tangential derivative of pressure. Indeed, the approximate azimuthal balances show that the net pressure gradient force (Fig. $16 \mathrm{~b}$ ) is more than an order of magnitude smaller than the Coriolis force. (Also note that the Coriolis force in the mean azimuthal balance is weaker by roughly a factor of five than in the radial balance.) The resulting geostrophic residual term is large and has the same form as the Coriolis term and therefore as the timemean radial velocity component. Of the remaining
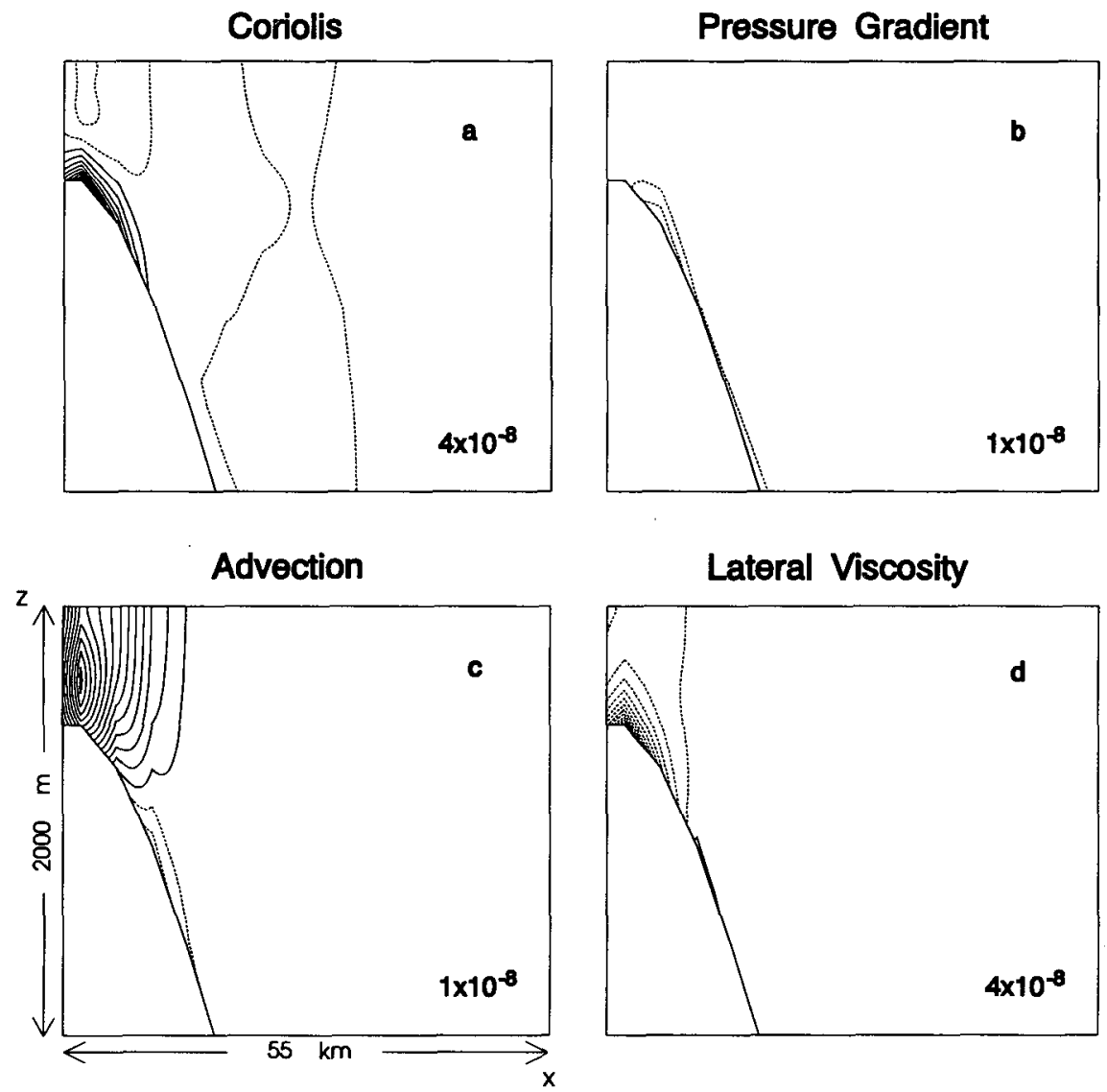

FIG. 16. Time-mean and azimuthally averaged momentum balance terms for the azimuthal component of velocity as a function of depth $(-2000 \mathrm{~m} \leqslant z \leqslant 0)$ and radial distance $(r \leqslant 55$ $\mathrm{km}$ ) from the seamount summit from experiment $3 \mathrm{~B}$ at $S=1.5$ : (a) Coriolis force, (b) pressure gradient force, (c) total advective terms ( mean plus eddy; horizontal plus vertical), and (d) lateral diffusion. $\mathrm{CI}$ as noted on the panels. 
terms, lateral viscosity (Fig. 16d) plays the dominant role in balancing the geostrophic residual. Note that viscous effects act to remove clockwise momentum from the upper flanks of the seamount. This viscous removal balances both the geostrophic residual term and the slow accumulation of clockwise momentum provided by horizontal eddy flux convergence (Fig. $16 \mathrm{c}$ ). The sum of terms in the time-mean azimuthal balance is again small.

The importance of viscous forces in the time-mean momentum balances is hardly surprising since some viscous process is necessary to achieve an equilibrium circulation. However, given the uncertainty over how to parameterize small-scale mixing processes, it is reassuring to note that the time-mean momentum cycle just described for experiment $3 \mathrm{~B}(S=1.5)$ is qualitatively similar if lateral viscosity is reduced in importance and Rayleigh damping substituted [experiment 3C $(S=1.5)]$. The resulting time-mean momentum balance in the azimuthal direction (Fig. 17) is again characterized by a large Coriolis force (Fig. 17a) associated with a radial flow outward along the flanks of the seamount. With no net (azimuthally averaged) pressure gradient force (Fig. 17b), the momentum balance is maintained by lateral advection of momentum (Fig. 17c) and by Rayleigh damping (Fig. 17d). The principal difference with the results of experiment $3 \mathrm{~B}$ is that net azimuthal momentum is extracted, rather than contributed, by horizontal eddy fluxes. The contribution of lateral viscosity is a factor of 5 smaller than that of the Rayleigh damping. The momentum cycle associated with the time-mean radial component of flow (not shown) is also qualitatively unaltered with Rayleigh damping.

Despite the degree of qualitative similarity seen in Figs. 16 and 17, there are some interesting differences as well. We note in particular that the residual circulation produced with Rayleigh damping is more highly bottom intensified than that which appears at higher levels of biharmonic lateral smoothing. The rather large rectification efficiency seen with Rayleigh damping, as large as $37 \%$ for experiments $3 \mathrm{C}$, is related to the ability of the fluid in the absence of strong lateral smoothing to concentrate net azimuthal momentum in a thin region surrounding the seamount. However, there is no indication of compensation between current strength
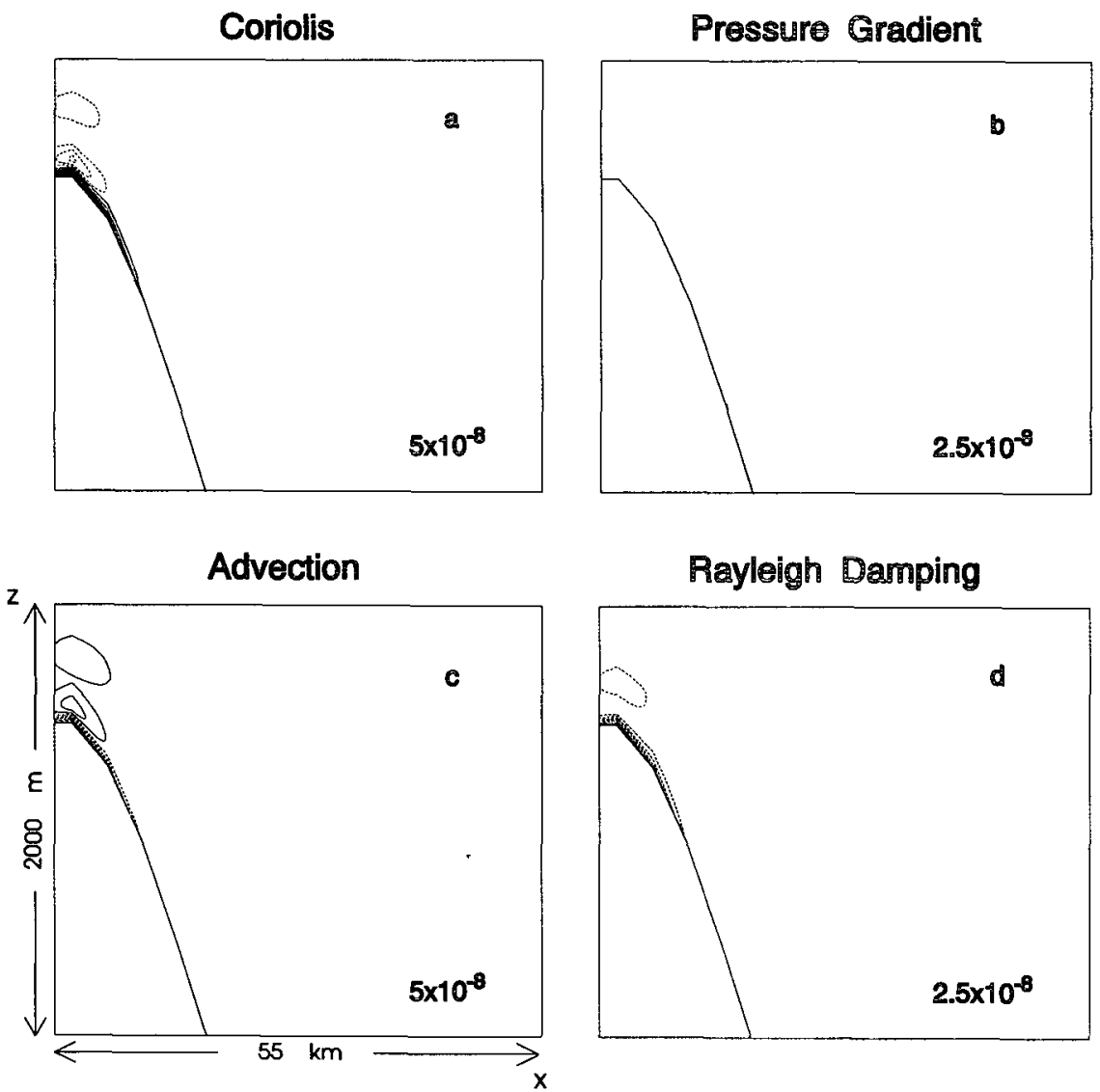

FIG. 17. Time-mean and azimuthally averaged momentum balance terms for the azimuthal component of velocity as a function of depth $(-2000 \mathrm{~m} \leqslant z \leqslant 0)$ and radial distance $(r \leqslant 55$ $\mathrm{km}$ ) from the seamount summit from experiment $3 \mathrm{C}$ at $S=1.5$ : (a) Coriolis force, (b) pressure gradient force, (c) total advective terms (mean plus eddy; horizontal plus vertical), and (d) lateral diffusion. $\mathrm{CI}$ as noted on the panels. 
and the thickness of the time-mean bottom boundary layer: the net azimuthal transport in experiment $3 \mathrm{C}$ is smaller by a factor of 10 than in experiment $3 \mathrm{~B}$.

\section{f. Comparison with prior theory}

Topographic rectification of tidal currents in the coastal oceans has been addressed analytically by a variety of authors beginning with the barotropic theories of Huthnance (1973) and Loder (1980). In these early studies, a rectification mechanism involving continuity and Coriolis effects, but regulated by bottom friction, was demonstrated and found to be strong enough to produce residual currents of strength comparable to those observed (e.g.) on the sides of Georges Bank. In this mechanism, alongisobath mean flows are generated by the Coriolis force in response to cross-isobath (radial) particle displacements. However, radial wave motions are asymmetric in the presence of bottom stress effects, since the latter are weaker for outwardly directed flow. The introduction of bottom stress therefore breaks the inherent symmetry of the inviscid nonlinear equations, and allows the production of net mean flow about the seamount.

The more recent theoretical contributions of Wright and Loder (1985) and Maas and Zimmerman $(1989 a, b)$ have reexamined the issue of nonlinear generation of residual currents with particular attention to the vertical structure of the residual currents, and the roles of friction and stratification, respectively. Wright and Loder assumed a homogeneous fluid, finite amplitude topography, and a frictional parameterization combining vertical viscosity with a linear bottom drag law. Maas and Zimmerman explored the response of a uniformly stratified fluid to weak bottom variations in the presence of a spatially uniform modal damping term similar to our Rayleigh damping. Both studies assumed weak nonlinearity.

Despite the differences in formulation between these two studies, together they do portray a consistent picture of the residual circulation. In particular, the primary circulation is expected to be anticyclonic and bottom intensified, though with vertical structure dependent on the details of stratification and the frictional processes employed. Further, both studies suggest that the deep flow should be radially outward from the topographic feature. For our studies, these results suggest downwelling above the seamount. (Wright and Loder find these results only in the limit of "weak" friction which we would argue is ours as well.) The numerical experiments here demonstrate that these properties of the residual flow persist into the stratified, finite amplitude topography, three-dimensional, nonlinear regime, and that they are qualitatively (though not quantitatively) insensitive to frictional parameterization.

Lastly, we note a final point of comparison and possible disagreement with Wright and Loder (1985). Their analysis shows some tendency, but no universal argument, for cross-isobath bottom currents to be down the mean cross-isobath pressure gradient. In contrast, in the nonlinear runs for which we have produced mean momentum balances we always find a high correlation between bottom-intensified outward radial motion and the outwardly directed pressure gradient. The ratio of these two terms has never been observed to change sign nor to deviate in value by more than a factor of 2 . Whether this more promising result is a property of the fully nonlinear, finite amplitude limit or merely an artifact of an overly narrow parametric exploration cannot be settled by our limited number of experiments.

\section{Summary and discussion}

Using the semispectral, primitive equation ocean circulation model of Haidvogel et al. (1991b), we have revisited the problem of the resonant generation of seamount-trapped waves by weak oscillatory ambient currents, and the closely associated issue of the production of residual circulation about the seamount. The idealized context of the present study has been a tall, circular, isolated seamount in an initially resting ocean with fixed background stratification. The forcing is provided by an oscillatory barotropic current with no horizontal shear. Specific attention has been focused on the parametric sensitivity of the resonance and rectification mechanisms to changes in the vertical dependence and strength of the stratification, as well as to alternate subgrid-scale viscous closure assumptions.

The intent has been twofold. First, we have sought to extend prior analytical and numerical studies-limited to weak nonlinearity, small amplitude topography, or both-into the fully nonlinear regime, and to deduce and dynamically interpret the structure and strength of the residual circulation. Second, we seek (eventually) to more thoroughly account for the observed currents at abrupt topographic features such as Fieberling Guyot.

Numerical solutions obtained with the linearized versions of the equations of motion correspond closely to analytical results obtained previously by Chapman (1989) and Brink (1990). In particular, resonantly generated trapped waves of significant amplitude are found to occur broadly in parameter space; a precise match between the frequency of the imposed incident current and the frequency of the trapped free wave is not necessary to produce substantial excitation of the trapped wave. For horizontally uniform incident flow, the lowest mode seamount-trapped waves are preferentially excited.

The time-mean circulation about the seamount is dominated by a strong bottom-intensified, clockwise circulation closely trapped to the seamount. The transport carried by the residual circulation is a significant fraction of a Sverdrup for the central parameters used here. Maximum local time-mean current amplitudes are found to be as large as $37 \%$ of the magnitude of the propagating waves. In addition to the strong anti- 
cyclonic residual flow, however, we find a weaker (but perhaps equally important ) circulation in the verticalradial plane. This secondary circulation is characterized by persistent downwelling over the top of the seamount at all depths. Maximum vertical downwelling rates of several tens of meters per day occur at the summit of the seamount. The vertical mass flux accompanying this systematic downwelling is balanced by a slow radial flux of mass directed outward from the seamount.

Time-mean budgets for the radial and azimuthal components of momentum have been constructed to identify the dynamical balances that maintain the residual circulation. The resulting balances show that horizontal eddy fluxes of momentum are responsible for exchanging net radial and azimuthal momentum between the far field and the upper flanks of the seamount. There, Coriolis and pressure gradient forces provide the dominant balances in the radial direction. However, Coriolis and viscous forces jointly balance horizontal eddy fluxes for the azimuthal component of the residual currents.

Although our long-term goal is to produce regional models of sufficient realism as to be directly comparable with observations, the simplified aspects of the model studied here preclude such a direct comparison. Much potentially important dynamics is missing, including the generation of mean circulation by net correlation between bottom pressure and topographic roughness (i.e., topographic form stress), and the effects of horizontal, vertical, and temporal variability in the ambient currents (i.e., the effects of mesoscale eddies). These processes need further study.

Even so, this study supports prior conjecture that the resonant enhancement of trapped waves should be a relatively common phenomenon, and that the efficiency of the generation process should be capable of accounting for the magnitudes of the observed diurnal currents at locations such as Fieberling Guyot. The effectiveness of the production mechanism for residual circulation in our solutions, judged by mean flow strength as a fraction of wave amplitude, is less likely to account for all of the mean flow signals observed at Fieberling [perhaps as large as 50\%; see Eriksen (1991)]. (However, many parametric changes which enhance the resonance will strengthen the mean relative to the oscillatory flow.) Nonetheless, this does suggest that there may be additional dynamical mechanisms at work (e.g., eddy-topographic interactions) producing substantial local mean flows in the neighborhood of Fieberling.

In principle, "realistic" simulations of the timevarying and residual circulations about features such as Fieberling Guyot are possible. To carry out such simulations would require better estimates of several local environmental parameters such as the strength of the ambient tidal signal, and the space/time properties of the large-scale and mesoscale eddy fields. Estimates of all of these statistics can be obtained from observations made during the TOPO field program. A much more serious issue, likely to persist for the foreseeable future, is the need for better observations, theories, and ultimately parameterizations of small-scale mixing near steep topography. This is likely the ratedetermining step for realistic modeling of ocean circulation-topography interaction.

Acknowledgments. Support for this work was provided by the Office of Naval Research under Contracts NO-0014-89-J-1 106 and NO-0014-90-J-1044, and by the Institute for Naval Oceanography. The calculations reported herein were mainly conducted on a Stardent Titan computer system at the Institute of Marine and Coastal Sciences, Rutgers University. Thanks, as always, are due Kate Hedström for assistance in all phases of the study. Lastly, we are indebted to the reviewers for many constructive suggestions.

\section{REFERENCES}

Arakawa, A., and V. R. Lamb, 1977: Computational design of the basic dynamical processes of the UCLA General Circulation Model. Methods of Computational Physics, Vol. 17, Academic Press, 174-265.

Beckmann, A., and D. B. Haidvogel, 1993: Numerical simulation of flow around a tall isolated seamount. Part I: Problem formulation and model accuracy. J. Phys. Oceanogr., 23, 1736-1753.

Brink, K. H., 1989: The effect of stratification on seamount-trapped waves. Deep-Sea Res., 36, 825-844.

, 1990: On the generation of seamount-trapped waves. DeepSea Res., 37, 1569-1582.

Chapman, D. C., 1989: Enhanced subinertial diurnal tides over isolated topographic features. Deep-Sea Res., 36, 815-824.

- and D. B. Haidvogel, 1992: Formation of Taylor caps over a tall isolated seamount in a stratified ocean. Geophys. Astrophys. Fluid Dyn., 64, 31-65.

- and - , 1993: Generation of internal Lee waves trapped over a tall seamount. Geophys. Astrophys. Fluid Dyn., 69, 3354.

Eriksen, C. C., 1991: Observations of amplified flows atop a large seamount. J. Geophys. Res., 96, 15 227-15 236.

Genin, A., M. Noble, and P. F. Lonsdale, 1989: Tidal currents and anticyclonic motions on two North Pacific seamounts. DeepSea Res., 36, 1803-1815.

Haidvogel, D. B., A. Beckmann, and K. S. Hedström, 1991a: Dynamical simulations of filament formation and evolution in the Coastal Transition Zone. J. Geophys. Res., 96, 15 017-15 040.

, J. L. Wilkin, and R. E. Young, 1991b: A semi-spectral primitive equation ocean circulation model using vertical sigma and orthogonal curvilinear horizontal coordinates. J. Comput. Phys., 94, 151-185.

Haney, R. L., 1991: On the pressure gradient force over steep topography in sigma coordinate ocean models. J. Phys. Oceanogr., 21, 610-619.

Hunkins, K., 1986: Anomalous diurnal tidal currents on the Yermak Plateau. J. Mar. Res., 44, 51-69.

Huthnance, J. M., 1974: On the diurnal tidal currents over Rockall Bank. Deep-Sea Res., 21, 23-35.

Loder, J. W., 1980: Topographic rectification of tidal currents on the sides of Georges Bank. J. Phys. Oceanogr., 10, 1399-1416.

Maas, L. R. M., and J. T. F. Zimmerman, 1989a: Tide-topography interactions in a stratified shelf sea $I$. Basic equations for quasinonlinear internal tides. Geophys. Astrophys. Fluid Dyn., 45, $1-35$.

, and _ 1989b: Tide-topography interactions in a stratified shelf sea II. Bottom trapped internal tides and baroclinic residual currents. Geophys. Astrophys. Fluid Dyn., 45, 37-69.

Wright, D. G., and J. W. Loder, 1985: A depth-dependent study of the topographic rectification of tidal currents. Geophys. Astrophys. Fluid Dyn., 31, 169-220. 